Frequency Estimates for Aircraft Crashes into Nuclear Facilities at Los Alamos National Laboratory (LANL)

George D. Heindel 


\section{DISCLAIMER}

This report was prepared as an account of work sponsored by an agency of the United States Government. Neither the United States Government nor any agency thereof, nor any of their employees, make any warranty, express or implied, or assumes any legal liability or responsibility for the accuracy, completeness, or usefulness of any information, apparatus, product, or process disclosed, or represents that its use would not infringe privately owned rights. Reference herein to any specific commercial product, process, or service by trade name, trademark, manufacturer, or otherwise does not necessarily constitute or imply its endorsement, recommendation, or favoring by the United States Government or any agency thereof. The views and opinions of authors expressed herein do not necessarily state or reflect those of the United States Government or any agency thereof. 


\section{DISCLAIMER}

Portions of this document may be illegible electronic image products. Images are produced from the best available original document. 


\section{Contents}

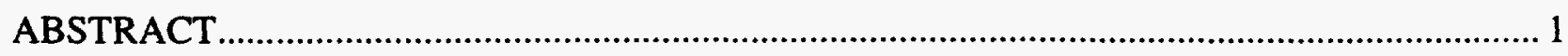

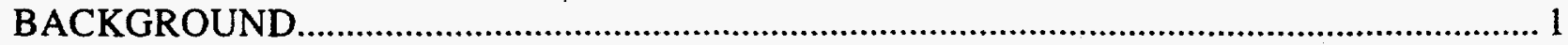

METHOD

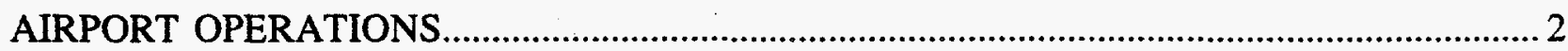

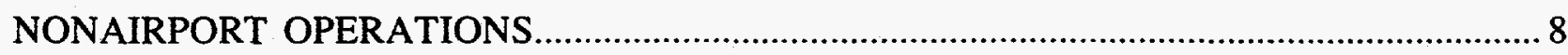

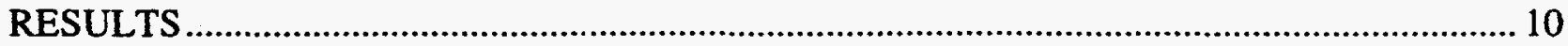

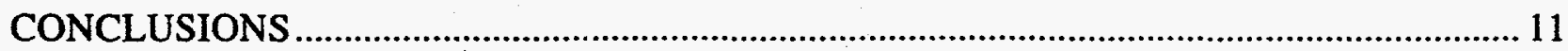

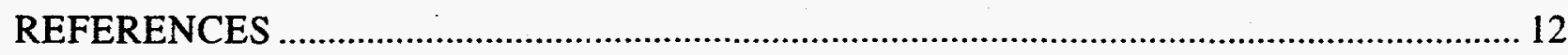

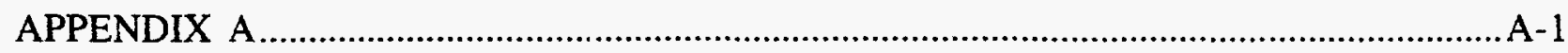

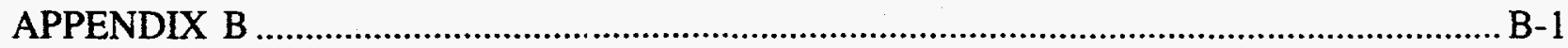

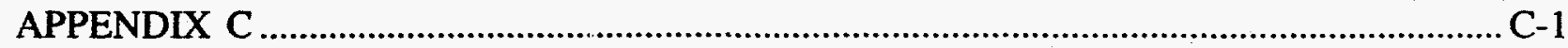

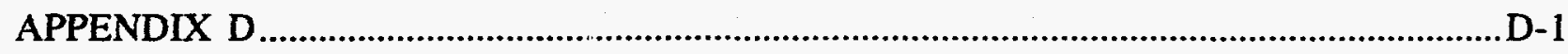

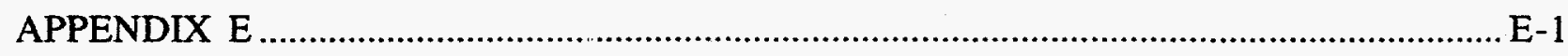

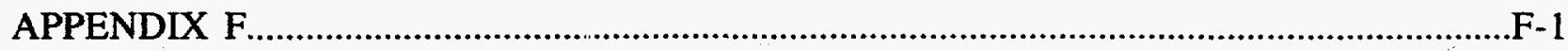

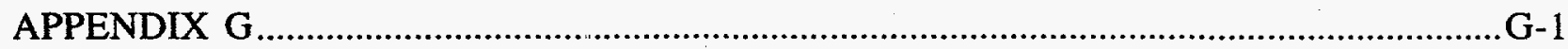

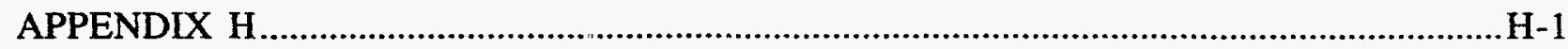

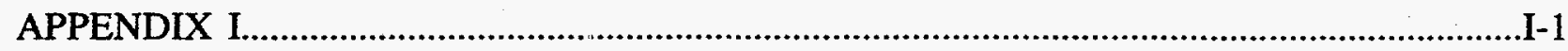

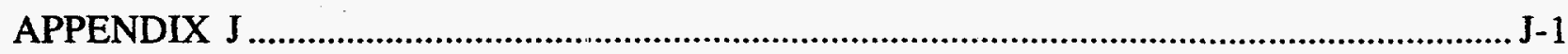

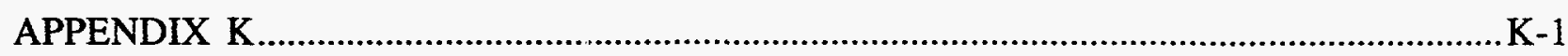

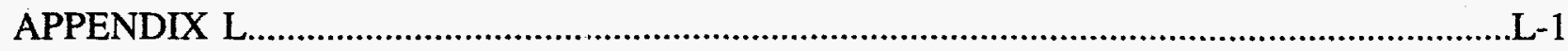

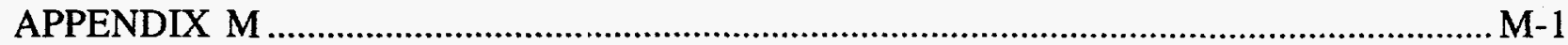




\title{
FREQUENCY ESTIMATES FOR AIRCRAFT CRASHES INTO NUCLEAR FACILITIES AT LOS ALAMOS NATIONAL LABORATORY (LANL)
}

by

\author{
George D. Heindel
}

\begin{abstract}
In October 1996, the Department of Energy (DOE) issued a new standard for evaluating accidental aircraft crashes into hazardous facilities. ${ }^{1}$ This document uses the method prescribed in the new standard to evaluate the likelihood of this type of accident occurring at Los Alamos National Laboratory's nuclear facilities.
\end{abstract}

\section{BACKGROUND}

According to DOE order and standard (DOE Order $5480.23^{2}$ and DOE-STD-3009-94 $4^{3}$ ), the likelihood of an airplane accidentally crashing into a nuclear facility must be considered in the safety analysis report (SAR) for that facility. If the likelihood is credible (generally defined as greater than $10^{-6} \mathrm{yr}^{-1}$ ), the consequences are evaluated and the scenario is included in the spectrum of accidents that make up the facility risk. The method that was used until now for estimating the likelihood of this scenario at Los Alamos is documented in an LA series report by Selvage. ${ }^{4}$ For each Los Alamos nuclear facility, this method was used to estimate the likelihood of an airplane crash occurring. In each case it was estimated to be less than the $10^{-6} \mathrm{yr}^{-1}$ limit of credibility, and so the consequences were not evaluated and the risks not considered as part of the facility risk. The method used was never endorsed by DOE. With the publication of DOE-STD-3014-94, there is now a DOE-prescribed method.

\section{METHOD}

The DOE-prescribed method for estimating the frequency of aircraft crashes into a given facility is described by the following four-factor formula [Equation (5-1) in DOE-STD-3014-96]:

$$
F=\sum_{i, j, k} N_{i j k} * P_{i j k} * f_{i j k}(x, y) * A_{i j}
$$

where 


$$
\begin{aligned}
& F=\quad \text { estimated annual aircraft crash impact frequency for the facility of interest } \\
& \text { (no./yr); } \\
& N_{i j k}=\quad \text { estimated annual number of site-specific aircraft operations (i.e., takeoffs, } \\
& \text { landings, and in-flights) for each applicable summation parameter (no./yr); } \\
& P_{i j k}=\quad \text { aircraft crash rate (per takeoff or landing for near-airport phases and per } \\
& \text { flight for the in-flight (nonairport) phase of operation for each applicable } \\
& \text { summation parameter; } \\
& f_{i j k}(x, y)=\text { aircraft crash location conditional probability (per square mile), given a } \\
& \text { crash, evaluated at the facility location for each applicable summation } \\
& \text { parameter; } \\
& A_{i j}=\quad \text { the site-specific effective area, including skid and fly-in effective areas } \\
& \text { (square miles), for the facility of interest for each applicable summation } \\
& \text { parameter, aircraft category or subcategory, and flight phase for military } \\
& \text { aviation; } \\
& i=\quad \text { (index for flight phases): } i=1,2 \text {, and } 3 \text { (takeoff, in-flight, and landing); } \\
& j=\quad \text { (index for aircraft category or subcategory): } j=1,2, \ldots, 11 \text {; } \\
& k=\quad \text { (index for flight source): } k=1,2, \ldots, \mathrm{K} \text { (possible multiple runways and } \\
& \text { nonairport operations); and } \\
& \sum_{i, j, k}=\sum_{k} \sum_{j} \sum_{i}=\text { site-specific summation over flight phase } i \text {; aircraft category or } \\
& \text { subcategory } j \text {; and flight source } k \text {. }
\end{aligned}
$$

In Los Alamos there is one airport, which has a single runway that is used by both general and commercial aviation (an air taxi service). In-flight operations are considered for general, commercial, and military aviation.

\section{AIRPORT OPERATIONS}

\section{Number of Aircraft Operations $N$}

The number of airport operations (both takeoffs and landings) at the Los Alamos airport was taken from a Los Alamos report ${ }^{4}$ that used data from the airport log for 1993. These data were qualitatively confirmed in 1997. During 1993 there were a total of 3600 commercial flight takeoffs and landings and 8834 general aviation takeoffs and landings.

The value of $N$ for in-flight crashes is collapsed into a combined $N P f(x, y)$ parameter provided by DOE-STD-3014-96. The in-flight (nonairport) method is described below. 


\section{Aircraft Crash Rate $\boldsymbol{P}$}

Aircraft crash rates by aircraft category, subcategory, and flight phase are provided in Table 1, which was taken from DOE-STD-3014-96, Table B-1. The value of $P$ for in-flight crashes is collapsed into a combined $N P f(x, y)$ parameter, also provided by the DOE standard. The in-flight method is described below.

Table 1. Aircraft Crash Rates by Category, Subcategory, and Flight Phase

\begin{tabular}{|c|c|c|}
\hline AlRCRAFT & \multicolumn{2}{|c|}{ CRASH RATE } \\
\hline General Aviation & Crashes per takeoff & Crashes per landing \\
\hline $\begin{array}{c}\text { 1. Fixed-Wing Single- } \\
\text { Engine Reciprocating }\end{array}$ & $1.1 \mathrm{E}-5$ & $2.0 \mathrm{E}-5$ \\
\hline $\begin{array}{c}\text { 2. Fixed-Wing Multiengine } \\
\text { Reciprocating }\end{array}$ & $9.3 \mathrm{E}-6$ & $2.3 \mathrm{E}-5$ \\
\hline 3. Fixed-Wing Turboprop & $3.5 \mathrm{E}-6$ & $8.3 \mathrm{E}-6$ \\
\hline 4. Fixed-Wing Turbojet & $1.4 \mathrm{E}-6$ & $4.7 \mathrm{E}-6$ \\
\hline Representative Fixed-Wing & $1.1 \mathrm{E}-5$ & $2.0 \mathrm{E}-5$ \\
\hline Representative Helicopter & $2.5 \mathrm{E}-5$ & \\
\hline Commercial & & $2.8 \mathrm{E}-7$ \\
\hline 1. Air Carrier & $1.9 \mathrm{E}-7$ & $2.3 \mathrm{E}-6$ \\
\hline 2. Air Taxi & $1.0 \mathrm{E}-6$ & $1.6 \mathrm{E}-6$ \\
\hline Military & & $3.3 \mathrm{E}-6$ \\
\hline 1. Large Aircraft & $5.7 \mathrm{E}-7$ & \\
\hline 2. Small Aircraft & $1.8 \mathrm{E}-6$ & \\
\hline
\end{tabular}

\section{Aircraft Crash Location Conditional Probability $f_{i j k}(x, y)$}

Given that a crash occurs upon takeoff or landing (with frequency $P$ from Table 1), the DOE standard provides the spatial distribution of ground impacts in units of $\mathrm{mi}^{-2}$ as a function of location with respect to the airport $f_{i j k}(x, y)$ for each type of activity and aircraft ( $i$ and $j$ ). These numbers are in a matrix format with the matrix cells' sides each being one mile in length. There is a unique matrix for each type of aircraft and activity (takeoff or landing). Location with respect to the airport is designated as the orthonormal distance to the facility from the middle of the runway in standard Cartesian coordinates ( $x$ and $y$ ). The positive $x$ direction of the coordinate system is defined as being the direction of the activity (takeoff or landing). The positive $y$ direction relative to $x$, then, is determined by the right-hand rule. The relevant matrices for the work of this report are

- Table 2: Crash Location Probability $f(x, y)$ for Commercial Aircraft Takeoff

- Table 3: Crash Location Probability $f(x, y)$ for Commercial Aircraft Landing

- Table 4: Crash Location Probability $f(x, y)$ for General Aviation Aircraft Takeoff 
These tables are reproduced from DOE-STD-3014-96, Tables B-2 through B-5. The $f(x, y)$ values for nonairport (in-flight) operations are collapsed into a combined $N P f(x, y)$ parameter that is also provided by the DOE standard. The in-flight method is described below.

\section{Effective Area $A_{i j}$}

The effective area $A_{i j}$ is the ground-surface area surrounding a facility that, if crashed into by an unobstructed aircraft, would result in an impact on the facility (either by direct fly-in or by skid). Equations (2); (3), and (4), which describe the effective area, are taken from the DOE standard [Equations (B-3 through B-5)].

$$
A_{\text {eff }}=A_{f}+A_{s}
$$

where

$$
A_{f}=(W S+R) * H \cot \phi+\frac{2 * L * W * W S}{R}+L * W
$$

and

$$
A_{s}=(W S+R) * S
$$

where

$$
\begin{aligned}
& A_{f}=\text { effective fly-in area; } \\
& A_{s}=\text { effective skid area; } \\
& W S=\text { aircraft wingspan (see Table } 6) ; \\
& R=\quad \text { length of the diagonal of the facility }=\left(L^{2}+W^{2}\right)^{0.5} ; \\
& H=\quad \text { height of specific facility; } \\
& \cot \phi=\text { mean of the cotangent of the aircraft impact angle (provided in Table 7) (for in- } \\
& \quad \begin{array}{l}
\text { flight crashes use the takeoff mean of the cotangent of the impact angle, if } \\
L=\quad \text { length of specific facility; }
\end{array}
\end{aligned}
$$




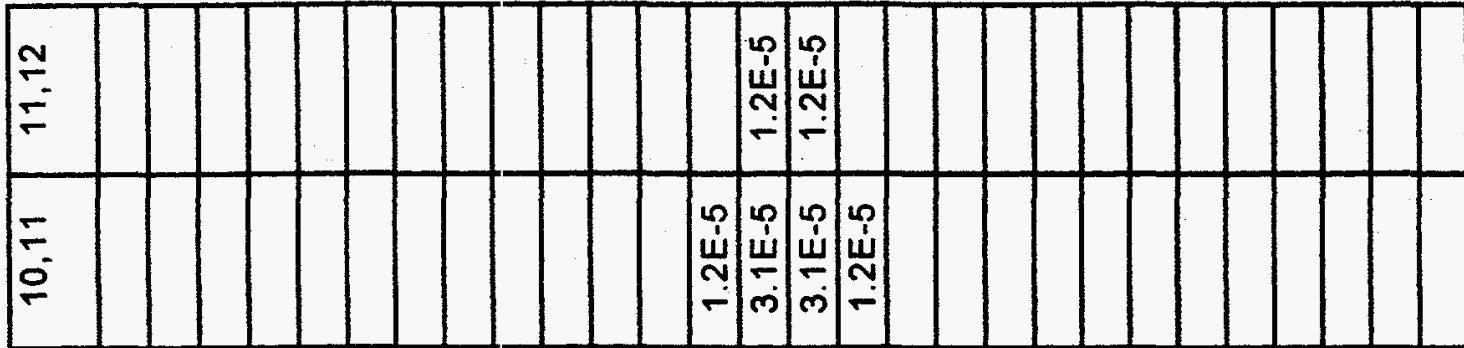

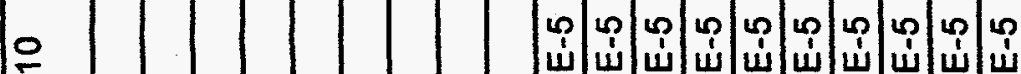

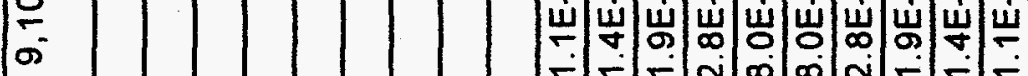

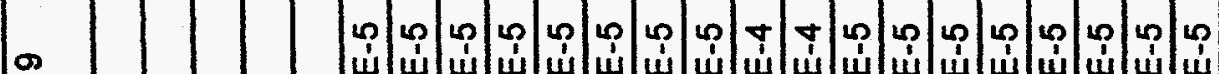

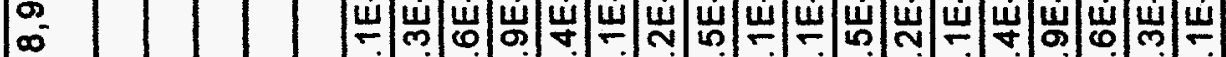

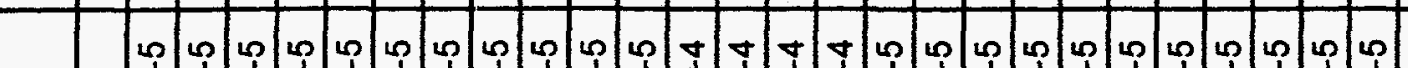

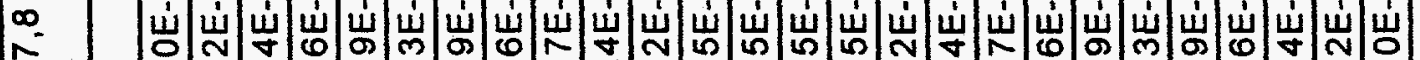

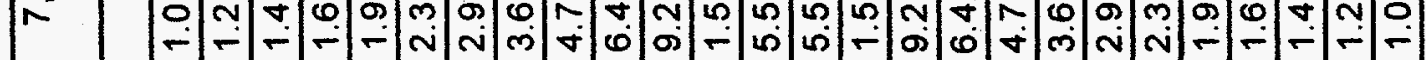

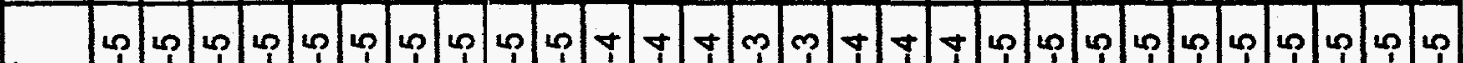

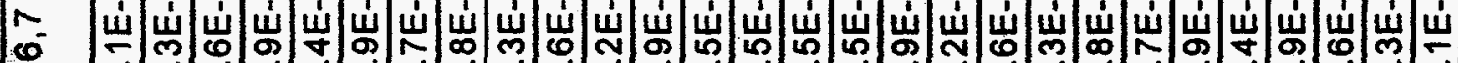

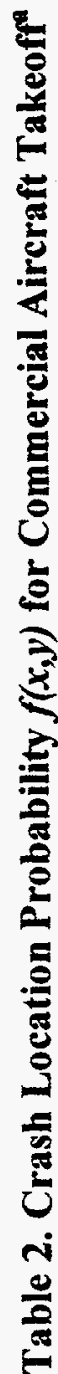

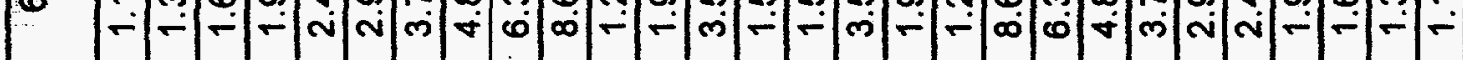

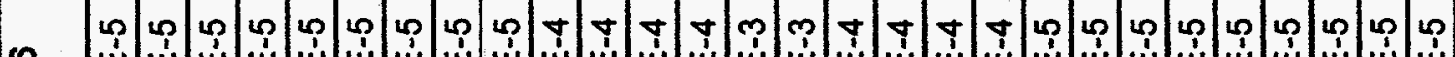

0 (

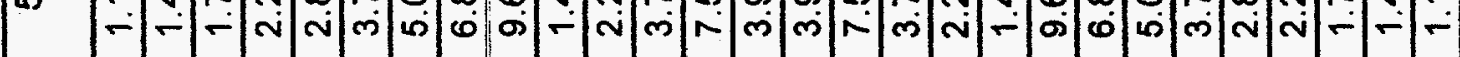

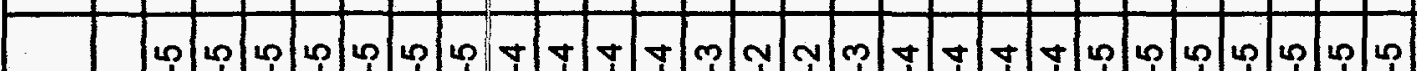

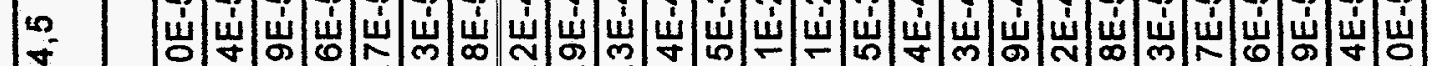

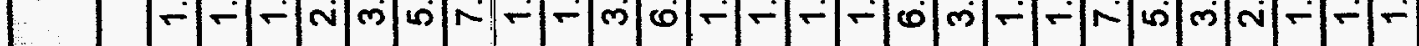

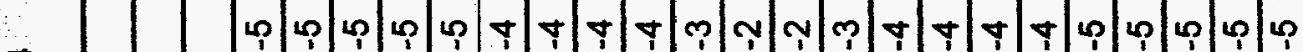

苟 Uே

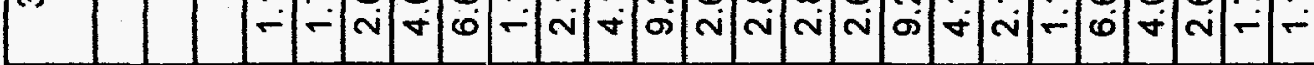

$m$

DL

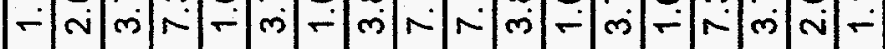

$\underline{-}$

L

Ш

-

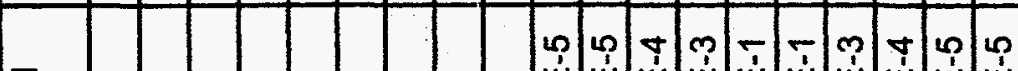

븐

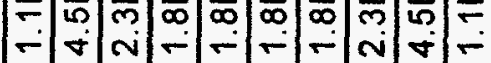

0

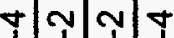

岩岗

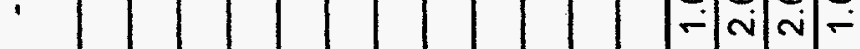

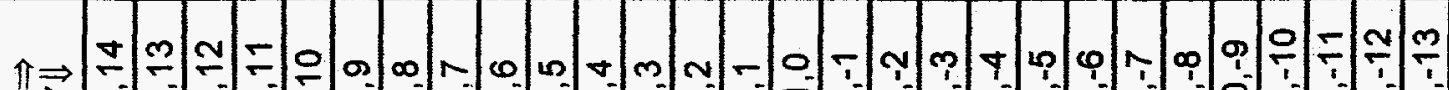

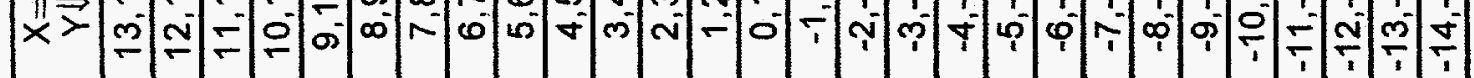



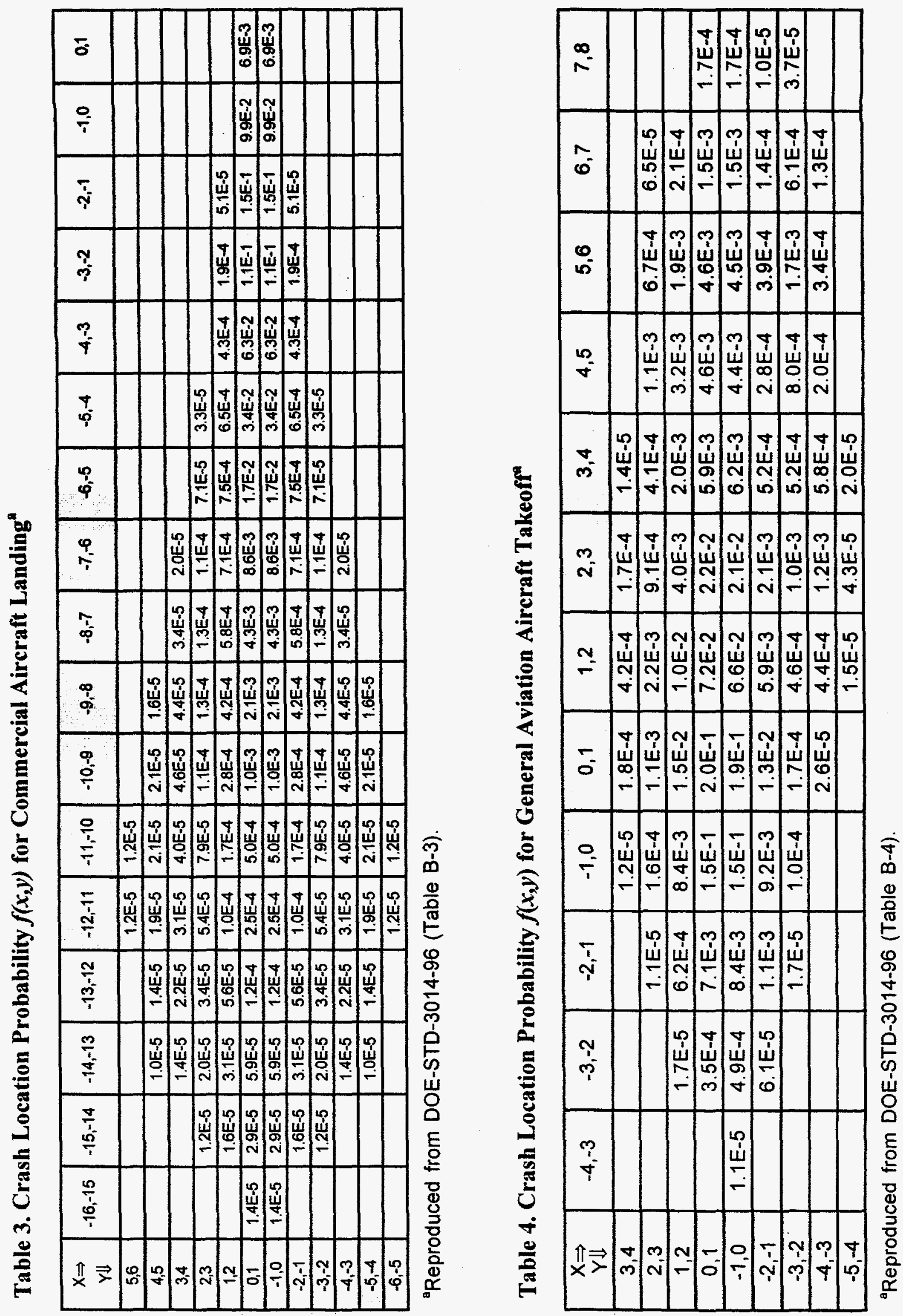


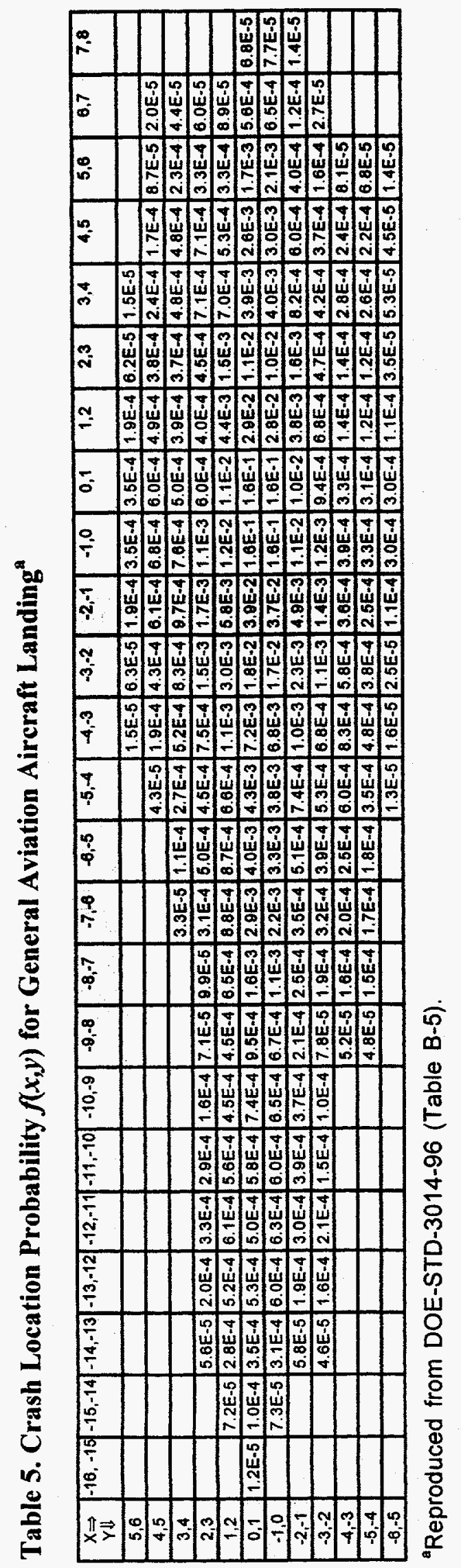


$W=$ width of specific facility; and

$S=\quad$ aircraft skid distance (mean value), provided in Table 8 (for in-flight crashes use the takeoff skid length, if available).

Facility dimensions were taken from the Selvedge report. ${ }^{4}$ Wingspans, impact angles, and skid distances, were taken from the DOE standard (Tables B-16, -17, and -18) and reproduced here as Tables 6, 7, and 8.

Table 6. Representative Wingspans (WS) for Commercial, General Aviation, and Military Aircraft

\begin{tabular}{|c|c|c|c|c|}
\hline General Aviation & Piston Engine & Turboprop & Turbojet & Helicopters \\
\hline \hline $50 \mathrm{ft}$ & $50 \mathrm{ft}$ & $73 \mathrm{ft}$ & $50 \mathrm{ft}$ & $50 \mathrm{ft}$ \\
\hline
\end{tabular}

\begin{tabular}{|c|c|c|}
\hline Commercial Aviation & Air Carrier & Air Taxi \\
\hline \hline & $98 \mathrm{ft}$ & $59 \mathrm{ft}$ \\
\hline
\end{tabular}

\begin{tabular}{|c|c|c|c|}
\hline Military Aviation & Large Aircraft & $\begin{array}{c}\text { Small Aircraft } \\
\text { High Performance }\end{array}$ & $\begin{array}{c}\text { Small Aircraft } \\
\text { Low Performance }^{\mathrm{b}}\end{array}$ \\
\hline & $223 \mathrm{ft}$ & $78 \mathrm{ft}$ & $110 \mathrm{ft}$ \\
\hline
\end{tabular}

Includes fighters, attackers, and trainers.

Includes other small aircraft.

Table 7. Values of the Mean of the Cotangent of the Impact Angle $(\cot \phi)$

\begin{tabular}{|c|c|c|c|c|c|c|c|}
\hline \multirow{2}{*}{$\begin{array}{c}\text { Aircraft } \\
\text { Category }\end{array}$} & \multirow{2}{*}{$\begin{array}{c}\text { Commercial } \\
\text { Aviation }\end{array}$} & \multirow{2}{*}{$\begin{array}{c}\text { General } \\
\text { Aviation }\end{array}$} & \multirow{2}{*}{ Helicopters } & & \multicolumn{3}{|c|}{ Military Aviation } \\
\cline { 5 - 8 } & & & Large Aircraft & \multicolumn{2}{|c|}{ Small Aircraft } \\
\hline \hline Mean $(\cot \phi)$ & 10.2 & 8.2 & 0.58 & 7.4 & 9.7 & 8.4 & 10.4 \\
\hline
\end{tabular}

Table 8. Mean Skid Distances ( $S$ ) for Each Aircraft Category

\begin{tabular}{|c|c|c|c|c|c|c|c|}
\hline \multirow{2}{*}{$\begin{array}{c}\text { Aircraft } \\
\text { Category }\end{array}$} & \multirow{2}{*}{$\begin{array}{c}\text { Commercial } \\
\text { Aviation }\end{array}$} & \multirow{2}{*}{$\begin{array}{c}\text { General } \\
\text { Aviation }\end{array}$} & \multirow{2}{*}{ Helicopters } & & \multicolumn{3}{|c|}{ Military Aviation } \\
\cline { 5 - 8 } & & & Large Aircraft & \multicolumn{2}{|c|}{ Small Aircraft } \\
\cline { 5 - 8 } \\
\hline $\begin{array}{c}\text { Mean Skid } \\
\text { Distance (ft) }\end{array}$ & 1440 & 60 & 0 & 780 & 368 & 246 & 447 \\
\hline
\end{tabular}

\section{NONAIRPORT OPERATIONS}

Aircraft crashes can occur as a result of in-flight problems not associated with takeoffs and landings at an airport. For our previous analytical method ${ }^{4}$ we determined an estimated impact 
frequency as a function of the distance of the facility from an established airway. Aircraft are no longer restricted to established airways but fly point-to-point. For this reason, the DOE standard suggests a different approach. By dividing the continental United States (CONUS) into regions; considering the amount of air traffic in that region; applying a base in-flight crash rate; and operating under the assumption that a crash is equally likely to occur at any area within that region, the Standard collapsed the first three terms in Equation (1) into a single term that is specific to each DOE site (e.g., Los Alamos). These values, which depend on both the location of the sites and the category of the aircraft, are presented in Tables 9 and 10. The values are then multiplied by the effective areas (in sq mi) of the facilities, providing the impact frequency from this operational phase.

Table 9. DOE Site-Specific Values and Maximum, Minimum, and Average Values for the Continental United States (CONUS) of $N P f(x, y)$ for General Aviation (GA) Nonairport Operation $s^{\mathbf{a}, \mathbf{b}}$

\begin{tabular}{|c|c|}
\hline Site & Value of NPf(x,y) \\
\hline \hline CONUS, Maximum & $3 \mathrm{E}-3$ \\
\hline CONUS, Minimum & $1 \mathrm{E}-7$ \\
\hline CONUS, Average & $2 \mathrm{E}-4$ \\
\hline Argonne National Laboratory & $3 \mathrm{E}-3$ \\
\hline Brookhaven National Laboratory & $5 \mathrm{E}-4$ \\
\hline Hanford & $1 \mathrm{E}-4$ \\
\hline Idaho National Engineering Laboratory & $9 \mathrm{E}-5$ \\
\hline Kansas City Plant & $6 \mathrm{E}-4$ \\
\hline Los Alamos National Laboratory & $2 \mathrm{E}-4$ \\
\hline Lawrence Livermore National Laboratory & $1 \mathrm{E}-4$ \\
\hline Mound & $4 \mathrm{E}-4$ \\
\hline Nevada Test Site & $8 \mathrm{E}-5$ \\
\hline Oak Ridge National Laboratory & $2 \mathrm{E}-3$ \\
\hline Fiantex & $7 \mathrm{E}-5$ \\
\hline Pinellas & $3 \mathrm{E}-4$ \\
\hline Rocky Flats & $2 \mathrm{E}-3$ \\
\hline Sandia National Labortories & $1 \mathrm{E}-3$ \\
\hline Savannah River Site & $2 \mathrm{E}-4$ \\
\hline
\end{tabular}

${ }^{a}$ These values are given in crashes per square mile, per year, that are centered at the site.

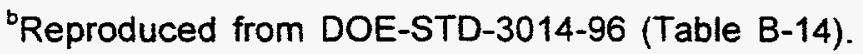


Table 10. DOE Site-Specific Values and Maximum, Minimum, and Average CONUS Values of $N P f(x, y)$ for Commercial and Military Aviation Nonairport Operations $^{\mathbf{a}, \mathbf{b}}$

\begin{tabular}{|c|c|c|c|c|}
\hline \multirow{2}{*}{ Site } & \multicolumn{3}{|c|}{ Values of NPf(x,y) } \\
\cline { 2 - 5 } & Air Carrier & Air Taxi & $\begin{array}{c}\text { Large } \\
\text { Military }\end{array}$ & $\begin{array}{c}\text { Small } \\
\text { Military }\end{array}$ \\
\hline \hline CONUS, Maximum & $2 \mathrm{E}-6$ & $8 \mathrm{E}-6$ & $7 \mathrm{E}-7$ & $6 \mathrm{E}-6$ \\
\hline CONUS, Minimum & $7 \mathrm{E}-8$ & $4 \mathrm{E}-7$ & $6 \mathrm{E}-8$ & $4 \mathrm{E}-8$ \\
\hline CONUS, Average & $4 \mathrm{E}-7$ & $1 \mathrm{E}-6$ & $2 \mathrm{E}-7$ & $4 \mathrm{E}-6$ \\
\hline Argonne National Laboratory & $7 \mathrm{E}-7$ & $4 \mathrm{E}-6$ & $9 \mathrm{E}-8$ & $8 \mathrm{E}-7$ \\
\hline Brookhaven National Laboratory & $2 \mathrm{E}-6$ & $8 \mathrm{E}-6$ & $7 \mathrm{E}-7$ & $2 \mathrm{E}-7$ \\
\hline Hanford & $1 \mathrm{E}-7$ & $1 \mathrm{E}-6$ & $1 \mathrm{E}-7$ & $4 \mathrm{E}-8$ \\
\hline Idaho National Engineering \\
Laboratory & $7 \mathrm{E}-8$ & $4 \mathrm{E}-7$ & $9 \mathrm{E}-8$ & $7 \mathrm{E}-7$ \\
\hline Kansas City Plant & $4 \mathrm{E}-7^{\mathrm{c}}$ & $1 \mathrm{E}-6 \mathrm{c}$ & $2 \mathrm{E}-7$ & $1 \mathrm{E}-6$ \\
\hline Los Alamos National Laboratory & $2 \mathrm{E}-7$ & $3 \mathrm{E}-6$ & $1 \mathrm{E}-7$ & $5 \mathrm{E}-6$ \\
\hline Lawrence Livermore National & $5 \mathrm{E}-7$ & $2 \mathrm{E}-6$ & $2 \mathrm{E}-7$ & $3 \mathrm{E}-6$ \\
\hline Laboratory & & & & \\
\hline Mound & $6 \mathrm{E}-7$ & $3 \mathrm{E}-6$ & $1 \mathrm{E}-7$ & $2 \mathrm{E}-6$ \\
\hline Nevada Test Site & $5 \mathrm{E}-7$ & $2 \mathrm{E}-6$ & $2 \mathrm{E}-7$ & $6 \mathrm{E}-6$ \\
\hline Oak Ridge National Laboratory & $6 \mathrm{E}-7$ & $2 \mathrm{E}-6$ & $1 \mathrm{E}-7$ & $6 \mathrm{E}-7$ \\
\hline Pantex & $2 \mathrm{E}-7$ & $3 \mathrm{E}-7$ & $1 \mathrm{E}-7$ & $5 \mathrm{E}-6$ \\
\hline Pinellas & $4 \mathrm{E}-7$ & $1 \mathrm{E}-6$ & $2 \mathrm{E}-7$ & $4 \mathrm{E}-6$ \\
\hline Rocky Flats & $2 \mathrm{E}-7$ & $6 \mathrm{E}-7$ & $9 \mathrm{E}-8$ & $9 \mathrm{E}-7$ \\
\hline Sandia National Laboratories & $2 \mathrm{E}-7$ & $3 \mathrm{E}-7$ & $1 \mathrm{E}-7$ & $5 \mathrm{E}-6$ \\
\hline Savannah River Site & $6 \mathrm{E}-7$ & $2 \mathrm{E}-6$ & $1 \mathrm{E}-7$ & $6 \mathrm{E}-7$ \\
\hline
\end{tabular}

${ }^{2}$ These values are given in crashes per square mile, per year, that are centered at the site.

${ }^{D}$ Reproduced from DOE-STD-3014-96 (Table B-15).

${ }^{c}$ The average CONUS was used for these entries.

\section{RESULTS}

Table 11 lists the total impact frequency estimates for each of the traditional nuclear facilities at Los Alamos, including airport and nonairport operations, takeoffs, landings, general aviation, commercial aviation, and military aviation. 
Table 11. Total Impact Frequency Estimates for Each of the Traditional Nuclear Facilities at Los Alamos

\begin{tabular}{|c|c|c|}
\hline Facility Name & Location & $\begin{array}{c}\text { Total Impact Frequency per } \\
\text { DoE-STD-3014-96 } \\
\text { (yr-1) }\end{array}$ \\
\hline \hline CMR & TA-3-29 & $1.2 \mathrm{E}-05$ \\
\hline WETF & TA-16-205 & $1.0 \mathrm{E}-06$ \\
\hline Hillside Vault & TA-18-26 & $1.1 \mathrm{E}-07$ \\
\hline LACEF Kiva 1 & TA-18-23 & $5.0 \mathrm{E}-07$ \\
\hline LACEF Kiva 2 & TA-18-32 & $5.3 \mathrm{E}-07$ \\
\hline LACEF Kiva 3 & TA-18-116 & $6.3 \mathrm{E}-07$ \\
\hline TSTA & TA-21-155 & $1.2 \mathrm{E}-04$ \\
\hline TSFF & TA-21-209 & $9.6 \mathrm{E}-05$ \\
\hline RLWTF & TA-50-1 & $8.3 \mathrm{E}-06$ \\
\hline TDF & TA-50-37 & $3.0 \mathrm{E}-06$ \\
\hline WCRRF & TA-50-69 & $1.6 \mathrm{E}-06$ \\
\hline Area G & TA-54-G & $3.8 \mathrm{E}-06$ \\
\hline Plutonium Facility & TA-55-4 & $5.6 \mathrm{E}-06$ \\
\hline
\end{tabular}

The tables in Appendixes A-M (one for each facility) provide the details of the calculations that resulted in the values shown in Table 11.

\section{CONCLUSIONS}

All LANL traditional nuclear facilities, except those at TA-18, have estimated aircraft crash impact frequencies that are greater than or equal to $10^{-6}$ per year according to the DOE-STD-3014-96 recommended method for calculating that frequency. The estimated frequency for WETF is very close to this limit, but those for others are significantly greater. The estimated frequency for WETF will increase approximately linearly with the planned increase in facility size.

Generally these results are dominated by the estimates for general aviation activity at the airport, and within that category, by crashes during landings.

As one might expect, the highest estimates are for the two facilities located at TA-21: TSTA and TSFF. These have total impact frequency estimates of $1.2 \mathrm{E}-04$ and $9.6 \mathrm{E}-05 \mathrm{yr}^{-1}$ respectively. While both commercial and general aviation contributions are greater than $10^{-6} \mathrm{yr}^{-1}$, the latter dominates the results.

These results suggest that future SARs and updates of old SARs should include consideration of this accident scenario. Consequence analyses are the logical next step for these facilities, and a standard approach for performing these analyses is also contained in DOE-STD-3014-96. 


\section{REFERENCES}

1. Accident Analysis for Aircraft Crash into Hazardous Facilities, DOE-STD-3014-96, U.S. Department of Energy, Washington, DC, October 1996.

2. Nuclear Safety Analysis Reports, DOE Order 5480.23, U.S. Department of Energy, Washington, DC, April 10, 1992.

3. Preparation Guide for U.S. Department of Energy Nonreactor Nuclear Facility Safety Analysis Reports, DOE-STD-3009-94, U.S. Department of Energy, Washington, DC, July 1994.

4. Selvage, Ronald D., Evaluation of Aircraft Crash Hazard at Los Alamos National Laboratory Facilities, Los Alamos National Laboratory report LA-13105, July 1996. 


\section{Appendix A}

\section{CMR (TA-3-29) Aircraft Crash Frequency Estimates}

Table A-1. Airport Operations (Takeoffs and Landings)

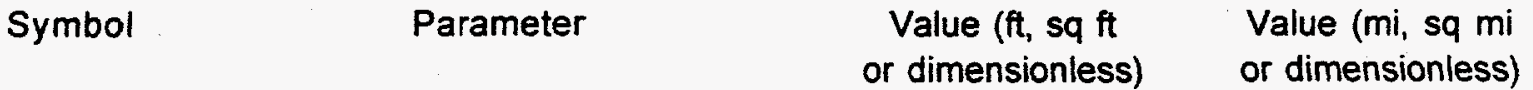

Facility Parameters

$x, y \quad$ Orthonormal distance from runway takeoff

$-2.66,-1.14$ landing

$L \quad$ Building length

$6.60 \mathrm{E}+02$

$+2.66,+1.14$

W Building width

$6.60 \mathrm{E}+02$

1.25E-01

$H \quad$ Building height

$5.00 \mathrm{E}+01$

1.25E-01

$R \quad$ Building diagonal

$9.33 \mathrm{E}+02$

9.47E-03

1.77E-01

Commercial Aircraft (Air Taxi) Calculations

$N \quad$ Number of flights per year (takeoffs + landings)

$3.6 E+03$

$P \quad$ Crash rate (Table 1)

per takeoff

per landing

1.0E-06

2.3E-06

$f(x, y) \quad$ Crash location probability takeoff (Table: 2) landing (Table 3)

$0.0 E+00$

$0.0 E+00$

WS Wingspan (Table 6)

59

Cot( $\phi)$ Cotangent of the crash angle

10.2

(Table 7)

$S \quad$ Skid distance (Table 8)

1440

$A_{f} \quad$ Effective fly-in area [Eq. (4)]

$A_{s} \quad$ Effective skid area [Eq. (5)]

$9.97 E+05$

3.58E-02

$1.43 E+06$

5.13E-02

$A_{\text {eff }} \quad$ Effective target area [Eq. (3)]

$F \quad$ Impact frequency (per yr) [Eq.

$2.43 E+06$

8.70E-02

takeoffs

(1)]

$0.0 E+00$

landings

$0.0 E+00$

total

$0.0 E+00$ 
Table A-1. (cont.)

Symbol

Parameter

Value ( $\mathrm{ft}$, sq $\mathrm{ft}$

Value (mi, sq mi

General Aviation Aircraft Calculations

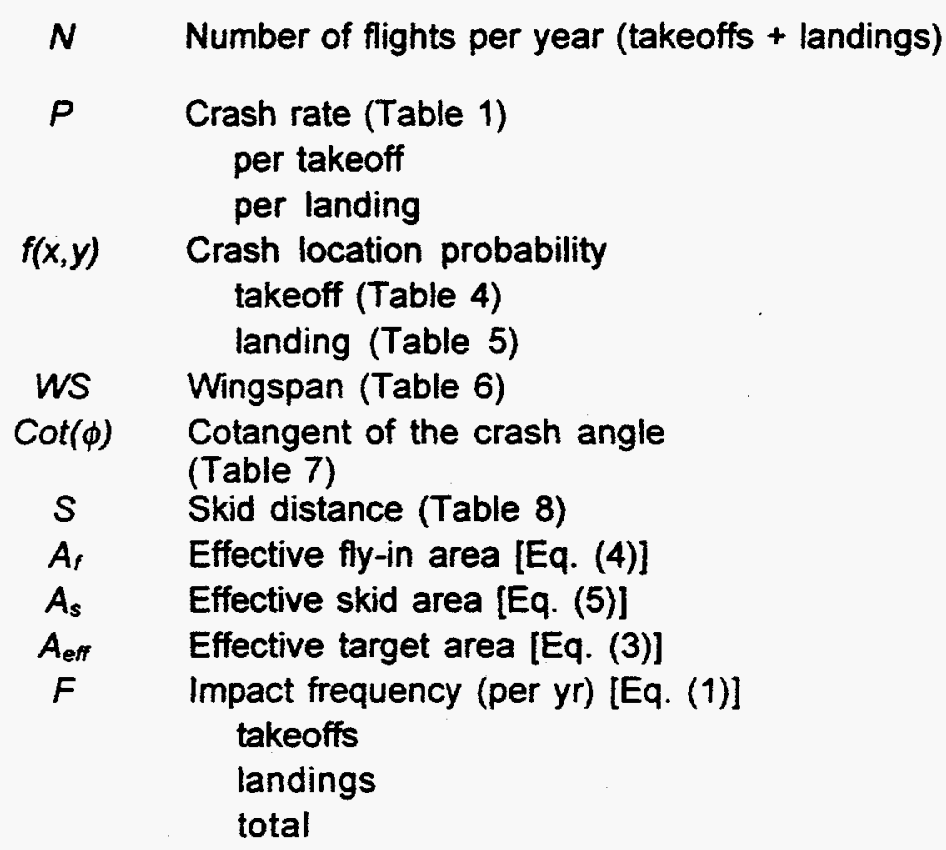

Total Impact Frequency from Airport Operations (per yr) 
Table A-2. Nonairport Operations (Overflights)

Symbol

Parameter

Value ( $\mathrm{ft}, \mathrm{sq} \mathrm{ft}$

Value (mi, sq mi or dimensionless)

NPf $(x, y) \quad$ Site-specific crash density rates

(Tables 9 and 10) (crashes per sq $\mathrm{mi}$

per yr)

commercial aviation (air taxi)

$3 E-06$

general aviation aircraft

$2 \mathrm{E}-04$

large military

$1 \mathrm{E}-07$

small military

$5 E-06$

\section{Effective Area Calculation for Military Aircraft}

\begin{tabular}{|c|c|c|}
\hline WS & $\begin{array}{l}\text { Wingspan (Table 6) } \\
\text { large aircraft } \\
\text { small aircraft }\end{array}$ & $\begin{array}{l}2.23 E+02 \\
1.10 E+02\end{array}$ \\
\hline $\operatorname{Cot}(\phi)$ & $\begin{array}{l}\text { Cotangent of the crash angle } \\
\text { (Table 7) } \\
\text { large aircraft } \\
\text { small aircraft }\end{array}$ & \\
\hline$S$ & $\begin{array}{l}\text { Skid distance (Table 8) } \\
\text { large aircraft } \\
\text { small aircraft }\end{array}$ & $\begin{array}{l}7.80 E+02 \\
2.46 E+02\end{array}$ \\
\hline$A_{f}$ & $\begin{array}{l}\text { Effective fly-in area [Eq. (4)] } \\
\text { large aircraft } \\
\text { small aircraft }\end{array}$ & $\begin{array}{l}1.07 E+06 \\
9.76 E+05\end{array}$ \\
\hline$A_{s}$ & $\begin{array}{l}\text { Effective skid area [Eq. (5)] } \\
\text { large aircraft } \\
\text { small aircraft }\end{array}$ & $\begin{array}{l}9.02 E+05 \\
2.57 E+05\end{array}$ \\
\hline$A_{\text {eff }}$ & $\begin{array}{l}\text { Effective target area [Eq. (3)] } \\
\text { large aircraft } \\
\text { small aircraft }\end{array}$ & $\begin{array}{l}1.97 E+06 \\
1.23 E+06\end{array}$ \\
\hline$F$ & $\begin{array}{l}\text { Impact frequency (per yr) [Eq. (1)] } \\
\text { commercial aviation } \\
\text { general aviation } \\
\text { military (large) } \\
\text { military (small) }\end{array}$ & \\
\hline
\end{tabular}

3.84E-02

$3.50 E-02$

3.24E-02

$9.21 \mathrm{E}-03$

7.08E-02

$4.42 \mathrm{E}-02$

2.6E-07

$6.8 \mathrm{E}-06$

7.1E-09

2.2E-07

Total Impact Frequency from Nonairport Operations (per yr)

commercial + general aviation + military

Total Impact Frequency (per yr)

airport + nonairport operations 


\section{Appendix B}

\section{WETF (TA-16-205) Aircraft Crash Frequency Estimates}

Table B-1. Airport Operations (Takeoffs and Landings)

Symbol

Parameter

Value ( $\mathrm{ft}$, sq $\mathrm{ft}$

Value (mi, sq mi or dimensionless) or dimensionless)

Facility Parameters

$x, y \quad$ Orthonormal distance from runway takeoff

$-3.9,-3.6$ landing

$+3.9,+3.6$

$L \quad$ Building length

$1.05 E+02$

$1.99 \mathrm{E}-02$

W Building width

$7.00 \mathrm{E}+01$

1.33E-02

$H \quad$ Building height

$6.00 E+01$

1.14E-02

$R \quad$ Building diagonal

$1.26 \mathrm{E}+02$

2.39E-02

Commercial Aircraft (Air Taxi) Calculations

\begin{tabular}{|c|c|}
\hline$N$ & Number of flights per year (takeoffs + landings) \\
\hline$P$ & $\begin{array}{l}\text { Crash rate (Table 1) } \\
\text { per takeoff } \\
\text { per landing }\end{array}$ \\
\hline$f(x, y)$ & $\begin{array}{l}\text { Crash location probability } \\
\text { takeoff (Table 2) } \\
\text { landing (Table 3) }\end{array}$ \\
\hline WS & Wingspan (Table 6) \\
\hline $\begin{array}{c}\operatorname{Cot}(\phi) \\
s\end{array}$ & $\begin{array}{l}\text { Cotangent of the crash angle } \\
\text { (Table } 7 \text { ) } \\
\text { Skid distance (Table } 8 \text { ) }\end{array}$ \\
\hline $\begin{array}{c}A_{f} \\
A_{s} \\
A_{\text {eff }} \\
F\end{array}$ & $\begin{array}{l}\text { Effective fly-in area [Eq. (4)] } \\
\text { Effective skid area [Eq. (5)] } \\
\text { Effective target area [Eq. (3)] } \\
\text { Impact frequency (per yr) [Eq. (1)] } \\
\text { takeoffs } \\
\text { landings } \\
\text { total }\end{array}$ \\
\hline
\end{tabular}


Table B-1. (cont.)

Symbol

Parameter

General Aviation Aircraft Calculations

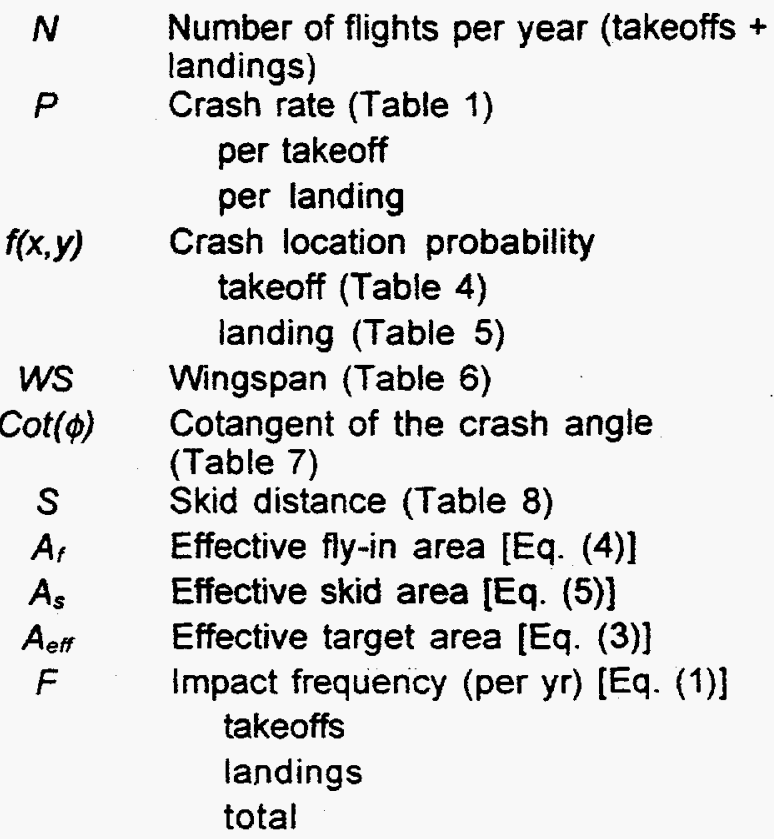

Total Impact Frequency from Airport Operations (per yr) 
Table B-2. Nonairport Operations (Overflights)

\begin{tabular}{|c|}
\hline Symbol \\
\hline
\end{tabular}

$$
\begin{aligned}
& \text { NPf(x,y) Site-specific crash density rates } \\
& \text { (Tables } 9 \text { and 10) (crashes per sq mi } \\
& \text { per yr) } \\
& \text { commercial aviation (air taxi) } \\
& \text { general aviation aircraft } \\
& \text { large military } \\
& \text { small military }
\end{aligned}
$$

\begin{tabular}{|c|c|c|}
\hline ws & $\begin{array}{l}\text { Wingspan (Table 6) } \\
\text { large aircraft } \\
\text { small aircraft }\end{array}$ & $\begin{array}{l}2.23 E+02 \\
1.10 E+02\end{array}$ \\
\hline $\operatorname{Cot}(\phi)$ & $\begin{array}{l}\text { Cotangent of the crash angle } \\
\text { (Table 7) } \\
\text { large aircraft } \\
\text { small aircraft }\end{array}$ & \\
\hline$s$ & $\begin{array}{l}\text { Skid distance (Table 8) } \\
\text { large aircraft } \\
\text { small aircraft }\end{array}$ & $\begin{array}{l}7.80 E+02 \\
2.46 E+02\end{array}$ \\
\hline$A_{f}$ & $\begin{array}{l}\text { Effective fly-in area [Eq. (4)] } \\
\text { large aircraft } \\
\text { small aircraft }\end{array}$ & $\begin{array}{l}1.88 \mathrm{E}+\mathrm{C} \\
1.39 \mathrm{E}+\mathrm{C}\end{array}$ \\
\hline$A_{s}$ & $\begin{array}{l}\text { Effective skid area [Eq. (5)] } \\
\text { large aircraft } \\
\text { small aircraft }\end{array}$ & $\begin{array}{l}2.72 \mathrm{E}+0.5 \\
5.81 \mathrm{E}+04\end{array}$ \\
\hline$A_{\text {eff }}$ & $\begin{array}{l}\text { Effective target area [Eq. (3)] } \\
\text { large aircraft } \\
\text { small aircraft }\end{array}$ & $\begin{array}{l}4.61 E+c \\
1.97 E+c\end{array}$ \\
\hline$F$ & $\begin{array}{l}\text { Impact frequency (per yr) [Eq. (1)] } \\
\text { commercial aviation } \\
\text { general aviation } \\
\text { military (large) } \\
\text { military (small) }\end{array}$ & \\
\hline
\end{tabular}

Effective Area Calculation for Military Aircraft

$6.76 \mathrm{E}-03$

4.99E-03

9.77E-03

$2.08 \mathrm{E}-03$

$1.65 \mathrm{E}-02$

7.08E-03

4.2E-08

$7.9 \mathrm{E}-07$

1.7E-09

$3.5 \mathrm{E}-08$

Total Impact Frequency from Nonairport Operations (per yr)

$$
\text { commercial + general aviation + military }
$$

Total Impact Frequency (per yr)

$$
\text { airport + nonairport operations }
$$




\section{Appendix C}

Hillside Vault (TA-18-26) Aircraft Crash Frequency Estimates

Table C-1. Airport Operations (Takeoffs and Landings)

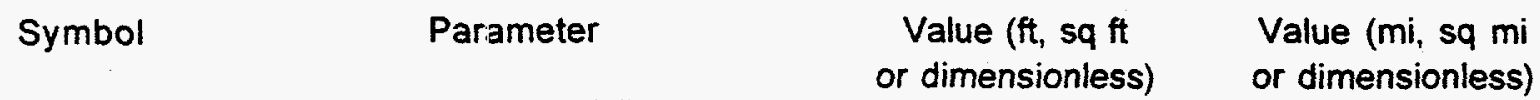

Facility Parameters

$(x, y)$ Orthonormal distance from runway takeoff

$+0.79,-2.5$

landing

$L \quad$ Building length

2.50E+01

$-0.79,+2.5$

$W \quad$ Building width

$2.50 E+01$

4.73E-03

$H \quad$ Building height

$0.00 \mathrm{E}+00$

4.73E-03

$R \quad$ Building diagonal

$3.54 E+01$

$0.00 E+00$

$6.70 \mathrm{E}-03$

Commercial Aircraft (Air Taxi) Calculations

$\begin{array}{cl}N & \text { Number of flights per year (takeoffs + landings) } \\ P & \text { Crash rate (Table 1) } \\ & \text { per takeoff } \\ & \text { per landing } \\ & \quad \begin{array}{l}\text { Crash location probability } \\ \text { takeoff (Table 2) }\end{array} \\ f(x, y) \quad \text { landing (Table 3) } \\ & \text { Wingspan (Table 6) } \\ W S & \text { Cotangent of the crash angle } \\ \text { Cot( } \phi) & \text { (Table 7) } \\ S & \text { Skid distance (Table 8) } \\ A_{f} & \text { Effective fly-in area [Eq. (4)] } \\ A_{s} & \text { Effective skid area [Eq. (5)] } \\ A_{\text {eff }} & \text { Effective target area [Eq. (3)] } \\ F & \text { Impact frequency (per yr) [Eq. (1)] } \\ & \text { takeoffs } \\ & \text { landings } \\ & \text { total }\end{array}$

$3.6 E+03$

1.0E-06

2.3E-06

2.3E-04

$0.0 E+00$

59

10.2

1440

$2.71 E+03$

9.72E-05

$1.36 E+05$

4.87E-03

$1.39 E+05$

4.97E-03

2.1E-09

$0.0 E+00$

2.1E-09 
Table C-1. (cont.)

Symbol

Parameter

Value $(\mathrm{ft}, \mathrm{sq} \mathrm{ft}$

Value (mi, sq mi

General Aviation Aircraft Calculations

$N \quad$ Number of flights per year (takeoffs + landings)

8.83E+03

P $\quad$ Crash rate (Table 1)

per takeoff

1.1E-05

per landing

2.0E-05

$f(x, y) \quad$ Crash location probability

takeoff (Table 4)

1.7E-04

landing (Table 5)

1.1E-03

WS Wingspan (Table 6)

50

$\operatorname{Cot}(\phi)$ Cotangent of the crash angle

8.2

(Table 7)

$S \quad$ Skid distance (Table 8)

$A_{f} \quad$ Effective fly-in area [Eq. (4)]

$A_{s} \quad$ Effective skid area [Eq. (5)]

$A_{\text {eff }} \quad$ Effective target area [Eq. (3)]

$F \quad$ Impact frequency (per yr) [Eq. (1)]

takeoffs

landings

60

2.39E+03

8.58E-05

$5.12 E+03$

1.84E-04

$7.51 E+03$

2.70E-04

2.2E-09

2.6E-08

total

2.8E-08

Total Impact Frequency from Airport Operations (per yr)

commercial + general aviation

3.0E-08 
Table C-2. Nonairport Operations (Overflights)

\begin{tabular}{|c|c|c|}
\hline Symbol & Parameter & $\begin{array}{c}\text { Value (ft, sq ft } \\
\text { or dimensionless) }\end{array}$ \\
\hline
\end{tabular}

NPf $(x, y) \quad$ Site-specific crash density rates

(Tables 9 and 10) (crashes per sq $\mathrm{mi}$

per yr)

commercial aviation (air taxi)

3E-06

general aviation aircraft

2E-04

large military

1E-07

small military

$5 E-06$

Effective Area Calculation for Military Aircraft

\begin{tabular}{|c|c|}
\hline WS & $\begin{array}{l}\text { Wingspan (Table 6) } \\
\text { large aircraft } \\
\text { small aircraft }\end{array}$ \\
\hline $\operatorname{Cot}(\phi)$ & $\begin{array}{l}\text { Cotangent of the crash angle } \\
\text { (Table 7) } \\
\text { large aircraft } \\
\text { small aircraft }\end{array}$ \\
\hline$S$ & $\begin{array}{l}\text { Skid distance (Table 8) } \\
\text { large aircraft } \\
\text { small aircraft }\end{array}$ \\
\hline$A_{f}$ & $\begin{array}{l}\text { Effective fly-in area [Eq. (4)] } \\
\text { large aircraft } \\
\text { small aircraft }\end{array}$ \\
\hline$A_{s}$ & $\begin{array}{l}\text { Effective skid area [Eq. (5)] } \\
\text { large aircraft } \\
\text { small aircraft }\end{array}$ \\
\hline$A_{\text {eff }}$ & $\begin{array}{l}\text { Effective target area [Eq. (3)] } \\
\text { large aircraft } \\
\text { small aircraft }\end{array}$ \\
\hline$F$ & $\begin{array}{l}\text { Impact frequency (per yr) [Eq. (1)] } \\
\text { commercial aviation } \\
\text { general avialion } \\
\text { military (large) } \\
\text { military (small) }\end{array}$ \\
\hline
\end{tabular}

2.23E+02

1.10E+02

7.4

8.4

$7.80 \mathrm{E}+02$

$2.46 \mathrm{E}+02$

$8.51 \mathrm{E}+03$

3.05E-04

$4.51 \mathrm{E}+03$

1.62E-04

$2.02 E+05$

7.23E-03

$3.58 \mathrm{E}+04$

1.28E-03

2. $10 E+05$

7.53E-03

$4.03 E+04$

1.44E-03

1.5E-08

5.4E-08

7.5E-10

7.2E-09

Total Impact Frequency from Nonairport Operations (per yr)

commercial + general aviation + military

7.7E-08

Total Impact Frequency (per yr)

airport + nonairport operations

1.1E-07 


\section{LACEF Kiva 1 (TA-18-23) Aircraft Crash Frequency Estimates}

\section{Table D-1. Airport Operations (Takeoffs and Landings)}

Symbol

Parameter

Facility Parameters

$\begin{array}{cl}(x, y) & \begin{array}{c}\text { Orthonormal distance from runway } \\ \text { takeoff } \\ \text { landing }\end{array} \\ L & \text { Building length } \\ W & \text { Building width } \\ H & \text { Building height: } \\ R & \text { Building diagonal }\end{array}$

6. $10 \mathrm{E}+01$

4.75E+01

$2.61 E+01$

$7.73 E+01$
$+0.59,-2.39$

$-0.59,+2.39$

1. $16 E-02$

$9.00 E-03$

4.94E-03

1.46E-02

Commercial Aircraft (Air Taxi) Calculations

\begin{tabular}{|c|c|}
\hline$N$ & Number of flights per year (takeoffs + landings) \\
\hline$P$ & $\begin{array}{l}\text { Crash rate (Tabile 1) } \\
\text { per takeoff } \\
\text { per landing }\end{array}$ \\
\hline$f(x, y)$ & $\begin{array}{l}\text { Crash location probability } \\
\text { takeoff (Table 2) } \\
\text { landing (Table 3) }\end{array}$ \\
\hline WS & Wingspan (Table 6) \\
\hline $\begin{array}{c}\operatorname{Cot}(\phi) \\
s\end{array}$ & $\begin{array}{l}\text { Cotangent of the crash angle } \\
\text { (Table 7) } \\
\text { Skid distance (Table } 8 \text { ) }\end{array}$ \\
\hline$A_{f}$ & Effective fly-in area [Eq. (4)] \\
\hline$A_{s}$ & Effective skid area [Eq. (5)] \\
\hline$A_{\text {eff }}$ & Effective target area [Eq. (3)] \\
\hline$F$ & $\begin{array}{l}\text { Impact frequency (per yr) [Eq. (1)] } \\
\text { takeoffs } \\
\text { landings } \\
\text { total }\end{array}$ \\
\hline
\end{tabular}


Table D-1. (cont.)

Symbol

Parameter

Value $(\mathrm{ft}, \mathrm{sq} \mathrm{ft}$

Value (mi, sq mi

or dimensionless)

or dimensionless)

General Aviation Aircraft Calculations

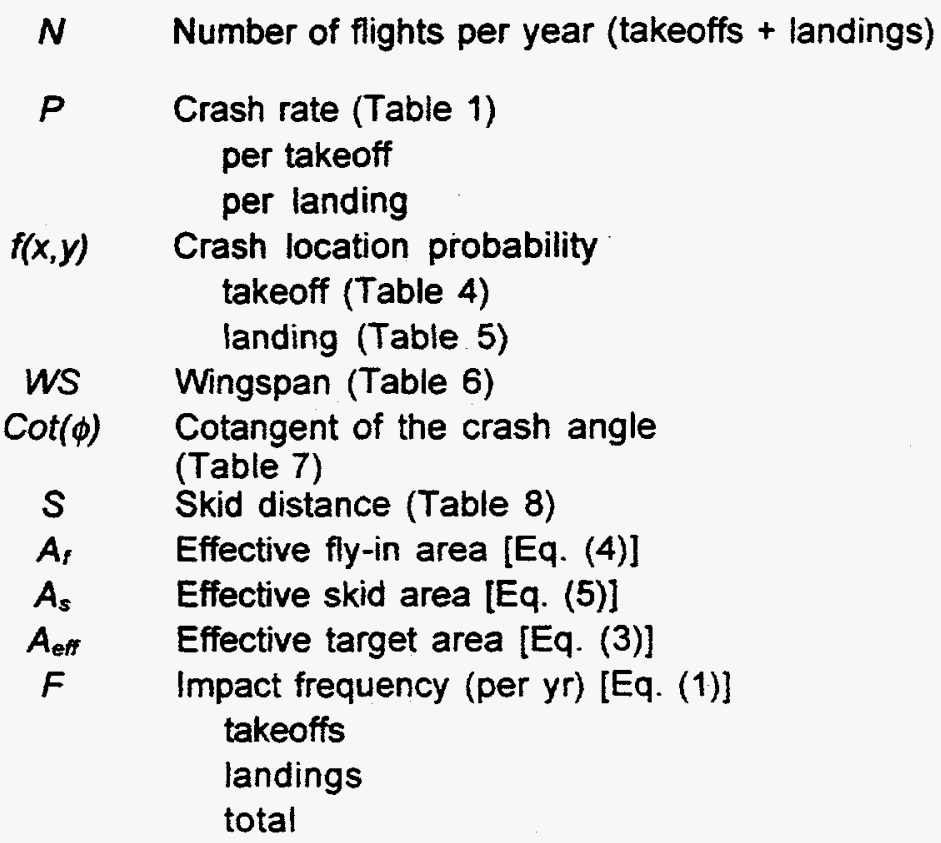

1.1E-05

2.0E-05

1.7E-04

1.1E-03

50

8.2

60

$3.39 \mathrm{E}+04$

1.22E-03

$7.64 E+03$

2.74E-04

$4.15 E+04$

1.49E-03

1.2E-08

$1.4 \mathrm{E}-07$

1.6E-07

Total Impact Frequency from Airport Operations (per yr)

commercial + general aviation

1.6E-07 
Table D-2. Nonairport Operations (Overflights)

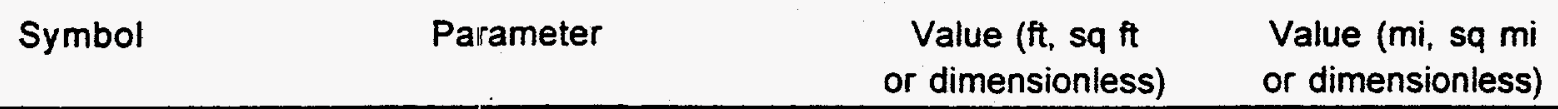

NPf $(x, y) \quad$ Site-specific crash density rates

(Tables 9 and 10) (crashes per sq $\mathrm{mi}$

per yr)

commercial aviation (air taxi)

3E-06

general aviation aircraft

$2 \mathrm{E}-04$

large military

$1 E-07$

small military

5E-06

Effective Area Calculation for Military Aircraft

\begin{tabular}{|c|c|}
\hline WS & $\begin{array}{l}\text { Wingspan (Table 6) } \\
\text { large aircraft } \\
\text { small aircraft }\end{array}$ \\
\hline $\operatorname{Cot}(\phi)$ & $\begin{array}{l}\text { Cotangent of the crash angle } \\
\text { (Table 7) } \\
\text { large aircraft } \\
\text { small aircraft }\end{array}$ \\
\hline$S$ & $\begin{array}{l}\text { Skid distance (Table 8) } \\
\text { large aircraft } \\
\text { small aircraft }\end{array}$ \\
\hline$A_{f}$ & $\begin{array}{l}\text { Effective fly-in area [Eq. (4)] } \\
\text { large aircraft } \\
\text { small aircraft }\end{array}$ \\
\hline$A_{s}$ & $\begin{array}{l}\text { Effective skid area [Eq. (5)] } \\
\text { large aircraft } \\
\text { small aircraft }\end{array}$ \\
\hline$A_{\text {eff }}$ & $\begin{array}{l}\text { Effective target area [Eq. (3)] } \\
\text { large aircraft } \\
\text { small aircraft }\end{array}$ \\
\hline$F$ & $\begin{array}{l}\text { Impact frequency (per yr) [Eq. (1)] } \\
\text { commercial aviation } \\
\text { general aviation } \\
\text { military (large) } \\
\text { military (small) }\end{array}$ \\
\hline
\end{tabular}

$2.23 E+02$

$1.10 E+02$

7.4

8.4

$7.80 \mathrm{E}+02$

$2.46 \mathrm{E}+02$

$7.76 E+04$

2.78E-03

$5.22 E+04$

1.87E-03

2. $34 E+05$

8. $40 E-03$

$4.61 E+04$

1.65E-03

3.12E+05

1.12E-02

$9.83 E+04$

3. $53 \mathrm{E}-03$

2.6E-08

3. $0 \mathrm{E}-07$

1.1E-09

1.8E-08

Total Impact Frequency from Nonairport Operations (per yr)

commercial + general aviation + military

3.4E-07

Total Impact Frequency (per yr)

airport + nonairport operations

5.0E-07 


\section{Appendix E}

\section{LACEF Kiva 2 (TA-18-32) Aircraft Crash Frequency Estimates}

Table E-1. Airport Operations (Takeoffs and Landings)

Symbol

Parameter

Value $(\mathrm{ft}$, sq $\mathrm{ft}$

Value (mi, sq mi or dimensionless) or dimensionless)

Facility Parameters

$(x, y) \quad$ Orthonormal distance from runway takeoff landing

$L \quad$ Building length

$5.85 E+01$

$5.75 E+01$

2.61E+01

$H \quad$ Building height

$8.20 E+01$

$+0.54,-2.64$

$-0.54,+2.64$

$R \quad$ Building diagonal

$1.11 \mathrm{E}-02$

1.09E-02

4.94E-03

1.55E-02

Commercial Aircraft (Air Taxi) Calculations

$\begin{array}{cl}N & \text { Number of flights per year (takeoffs + landings) } \\ P & \text { Crash rate (Table 1) } \\ & \text { per takeoff } \\ & \text { per landing } \\ & \text { Crash location probability } \\ & \text { takeoff (Table 2) } \\ f(x, y) \quad \text { landing (Table 3) } & \\ & \text { Wingspan (Table 6) } \\ W S & \text { Cotangent of the crash angle } \\ \text { Cot( } \phi) & \text { (Table 7) } \\ S & \text { Skid distance (Table 8) } \\ A_{f} & \text { Effective fly-in area [Eq. (4)] } \\ A_{s} & \text { Effective skid area [Eq. (5)] } \\ A_{\text {eff }} & \text { Effective target area [Eq. (3)] } \\ F & \text { Impact frequency (per yr) [Eq. (1)] } \\ & \text { takeoffs } \\ & \text { landings } \\ & \text { total }\end{array}$

1.0E-06

2.3E-06

2.3E-04

$0.0 E+00$ 
Talbe E-1. (cont.)

Symbol

Parameter

Value ( $\mathrm{ft}$, sq $\mathrm{ft}$ or dimensionless)
Value (mi, sq mi or dimensionless)

General Aviation Aircraft Calculations

$N \quad$ Number of flights per year (takeoffs + landings)

$8.83 E+03$

P $\quad$ Crash rate (Table 1)

per takeoff

1.1E-05

per landing

2.0E-05

$f(x, y) \quad$ Crash location probability takeoff (Table 4)

1.7E-04 landing (Table 5)

1.1E-03

WS Wingspan (Table 6)

Cot(phi) Cotangent of the crash angle

$S \quad$ Skid distance (Table 8)

$A_{f} \quad$ Effective fly-in area [Eq. (4)]

60

$A_{s} \quad$ Effective skid area [Eq. (5)]

$3.57 E+04$

1.28E-03

$A_{\text {eff }} \quad$ Effective target area [Eq. (3)]

$F$ Impact frequency (per yr) [Eq. (1)]

$7.92 \mathrm{E}+03$

2.84E-04

4. $36 E+04$

1.57E-03 takeoffs

1.3E-08 landings

$1.5 \mathrm{E}-07$ total

Total Impact Frequency from Airport Operations (per yr)

commercial + general aviation

1.7E-07 
Table E-2. Nonairport Operations (Overflights)

\begin{tabular}{|c|c|c|c|}
\hline Symbol & Farameter & $\begin{array}{l}\text { Value ( } \mathrm{ft}, \mathrm{sq} \mathrm{ft} \\
\text { or dimensionless) }\end{array}$ & $\begin{array}{l}\text { Value (mi, sq mi } \\
\text { or dimensionless) }\end{array}$ \\
\hline
\end{tabular}

$\operatorname{NPf}(x, y) \quad$ Site-specific crash density rates

(Tables 9 and 10) (crashes per sq mi

per yr)

commercial aviation (air taxi)

3E-06

general aviation aircraft

2E-04

large military

$1 E-07$

small military

5E-06

Effective Area Calculation for Military Aircraft

\begin{tabular}{|c|c|c|}
\hline WS & $\begin{array}{l}\text { Wingspan (Table 6) } \\
\text { large aircraft } \\
\text { small aircraft }\end{array}$ & $\begin{array}{l}2.23 E+0 \\
1.10 E+0\end{array}$ \\
\hline $\operatorname{Cot}(\phi)$ & $\begin{array}{l}\text { Cotangent of the crash angle } \\
\text { (Table } 7 \text { ) } \\
\text { large aircraft } \\
\text { small aircraft }\end{array}$ & $\begin{array}{l}7 . \\
8 .\end{array}$ \\
\hline$S$ & $\begin{array}{l}\text { Skid distance (Table 8) } \\
\text { large aircraft } \\
\text { small aircraft }\end{array}$ & $\begin{array}{l}7.80 E+0 \\
2.46 E+0\end{array}$ \\
\hline$A_{f}$ & $\begin{array}{l}\text { Effective fly-in area [Eq. (4)] } \\
\text { large aircraft } \\
\text { small aircraft }\end{array}$ & $\begin{array}{l}8.06 E+0 \\
5.45 E+0\end{array}$ \\
\hline$A_{s}$ & $\begin{array}{l}\text { Effective skid area [Eq. (5)] } \\
\text { large aircraft } \\
\text { small aircraft }\end{array}$ & $\begin{array}{l}2.38 E+0 \\
4.72 E+0\end{array}$ \\
\hline$A_{\text {eff }}$ & $\begin{array}{l}\text { Effective target area [Eq. (3)] } \\
\text { large aircraft } \\
\text { small aircraft }\end{array}$ & $\begin{array}{l}3.18 E+0 \\
1.02 E+0\end{array}$ \\
\hline$F$ & $\begin{array}{l}\text { Impact frequericy (per yr) [Eq. (1)] } \\
\text { commercial aviation } \\
\text { general aviation } \\
\text { military (large) } \\
\text { military (small) }\end{array}$ & \\
\hline
\end{tabular}

2.89E-03

$1.95 E-03$

8.53E-03

1.69E-03

1.14E-02

3.65E-03

2.7E-08

3.1E-07

1.1E-09

$1.8 E-08$

Total Impact Frequency from Nonairport Operations (per yr)

commercial + general aviation + military

3.6E-07

Total Impact Frequency (per yr)

airport + nonairport operations

5.3E-07 


\section{Appendix F}

\section{LACEF Kiva 3 (TA-18-116) Aircraft Crash Frequency Estimates}

\section{Table F-1. Airport Operations (Takeoffs and Landings)}

Symbol

Farameter

Value ( $\mathrm{ft}$, sq $\mathrm{ft}$

Value (mi, sq mi or dimensionless) or dimensionless)

Facility Parameters

$(x, y) \quad$ Orthonormal distance from runway takeoff $+0.85,-2.62$ landing

$-0.85,+2.62$

$L \quad$ Building length

8.10E+01

1.53E-02

$W \quad$ Building width

$6.40 E+01$

1.21E-02

$H \quad$ Building height

2.61E+01

4.94E-03

$R \quad$ Building diagonal

$1.03 E+02$

1.96E-02

Commercial Aircraft (Air Taxi) Calculations

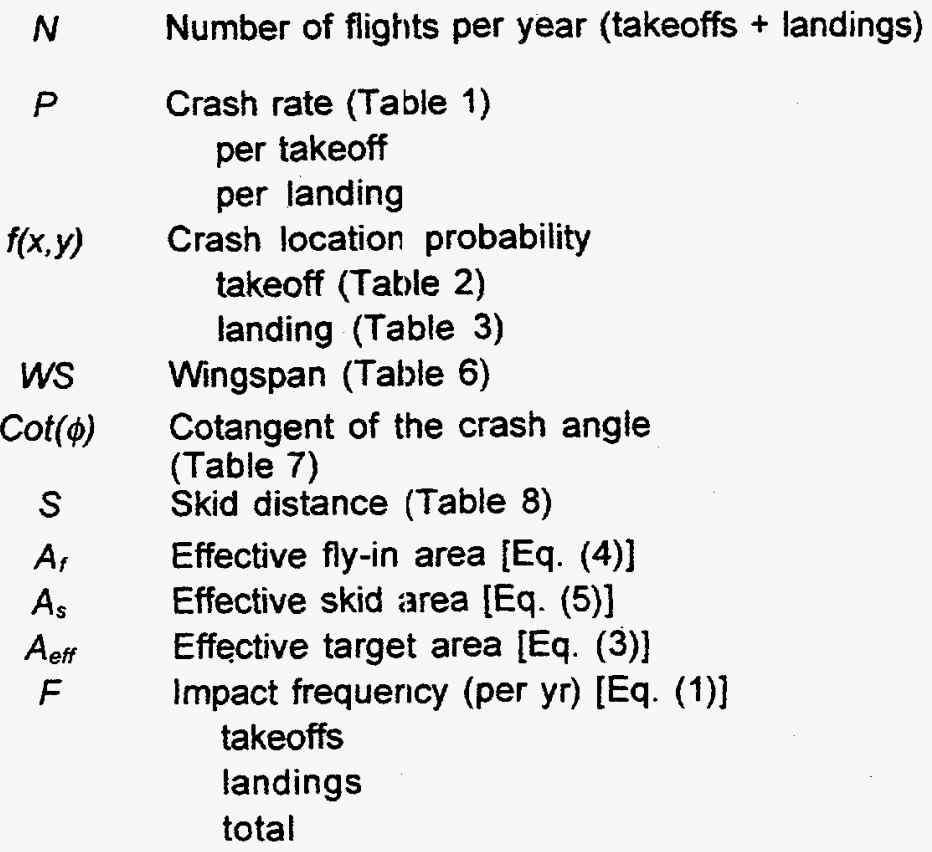


Table F-1. (cont.)

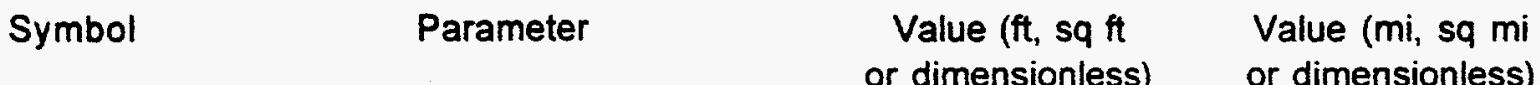

\section{General Aviation Aircraft Calculations}

$N \quad$ Number of flights per year (takeoffs + landings)

$8.83 E+03$

$P \quad$ Crash rate (Table 1)

per takeoff

1.1E-05

per landing

2.0E-05

$f(x, y) \quad$ Crash location probability takeoff (Table 4).

1.7E-04 landing (Table 5)

1.1E-03

WS Wingspan (Table 6)

$\operatorname{Cot}(\phi) \quad$ Cotangent of the crash angle (Table 7)

$S \quad$ Skid distance (Table 8)

$A_{f} \quad$ Effective fly-in area [Eq. (4)]

$A_{s} \quad$ Effective skid area [Eq. (5)]

$A_{\text {eff }} \quad$ Effective target area [Eq. (3)]

$F \quad$ Impact frequency (per yr) [Eq. (1)] takeoffs landings total

Total Impact Frequency from Airport Operations (per yr) 
Table F-2. Nonairport Operations (Overflights)

Symbol Parameter Value (ft, sq ft Value (mi, sq mi or dimensionless)

NPf $(x, y) \quad$ Site-specific crash density rates

(Tables 9 and 10) (crashes per sq $\mathrm{mi}$

per yr)

commercial aviation (air taxi)

$3 \mathrm{E}-06$

general aviation aircraft

$2 \mathrm{E}-04$

large military

$1 E-07$

small military

5E-06

Effective Area Calculation for Military Aircraft

WS Wingspan (Table 6)

large aircraft

$2.23 E+02$

small aircraft

$1.10 E+02$

Cot(phi) Cotangent of the crash angle

(Table 7)

large aircraft

small aircrait

S. Skid distance (Table 8)

large aircraft

$7.80 \mathrm{E}+02$

small aircrait

$2.46 \mathrm{E}+02$

$A_{f} \quad$ Effective fly-in area [Eq. (4)]

large aircraft

$9.06 \mathrm{E}+04$

small aircrait

$6.30 E+04$

3.25E-03

Effective skid area [Eq. (5)]

large aircraft

$2.54 E+05$

2.26E-03

small aircraft

$5.25 \mathrm{E}+04$

9.13E-03

Effective target area [Eq. (3)]

large aircraft

$3.45 E+05$

$1.88 \mathrm{E}-03$

$A_{\text {eff }}$

small aircraft

1. $15 E+05$

1.24E-02

4.14E-03

F Impact frequency (per yr) [Eq. (1)]

commercial aviation

3.1E-08

general aviation

3.7E-07

military (large)

1.2E-09

military (small)

2.1E-08

Total Impact Frequency from Nonairport Operations (per yr)

commercial + general aviation + military

Total Impact Frequency (per yr)

airport + nonairport operations

6.3E-07 


\section{Appendix G}

\section{TSTA (TA-21-155) Aircraft Crash Frequency Estimates}

\section{Table G-1. Airport Operations (Takeoffs and Landings)}

Symbol

Parameter

Value ( $\mathrm{ft}$, sq $\mathrm{ft}$ or dimensionless)
Value (mi, sq mi or dimensionless)

\section{Facility Parameters}

$(x, y)$ Orthonormal distance from runway takeoff landing

$L \quad$ Building length

$9.00 \mathrm{E}+01$

$-0.096,-0.26$

W Building width

$7.70 \mathrm{E}+01$

$9.80 \mathrm{E}+01$

$1.18 E+02$

$+0.096,+0.26$

$H \quad$ Building height

$1.70 E-02$

$1.46 \mathrm{E}-02$

$R \quad$ Building diagorlal

$1.86 \mathrm{E}-02$

2.24E-02

Commercial Aircraft (Air Taxi) Cialculations

N Number of flights per year (takeoffs + landings)

$3.6 E+03$

$P \quad$ Crash rate (Table 1)

per takeoff

.OE-0.6

per landing

2.3E-06

$f(x, y) \quad$ Crash location probability takeoff (Table 2)

2.6E-02

landing (Table 3)

6.9E-03

WS Wingspan (Table 6)

59

Cot $(\phi)$ Cotangent of the crash angle

(Table 7)

$S \quad$ Skid distance (Table 8)

6.86E-03

$A_{f} \quad$ Effective fly-in area [Eq. (4)]

$1.91 E+05$

$9.17 \mathrm{E}-03$

$A_{s} \quad$ Effective skid area [Eq. (5)]

$2.56 \mathrm{E}+05$

1.60E-02

$A_{\text {eff }} \quad$ Effective target area [Eq. (3)]

$F \quad$ Impact frequency (per yr) [Eq. (1)]

$4.47 E+05$

7.5E-07 takeoffs

4.6E-07

landings

$1.2 E-06$ 
Table G-1. (cont.)

Symbol

General Aviation Aircraft Calculations

$N \quad$ Number of flights per year (takeoffs + landings)

8.83E+03

$P \quad$ Crash rate (Table 1)

per takeoff

1.1E-05

per landing

2.0E-05

$f(x, y)$

Crash location probability takeoff (Table 4)

1.5E-01 landing (Table 5)

1.6E-01

WS Wingspan (Table 6)

$\operatorname{Cot}(\phi) \quad$ Cotangent of the crash angle

(Table 7)

$S \quad$ Skid distance (Table 8)

$A_{f} \quad$ Effective fly-in area [Eq. (4)]

$1.48 E+05$

5.31E-03

$A_{s} \quad$ Effective skid area [Eq. (5)]

$1.01 E+04$

3.63E-04

$A_{\text {eff }} \quad$ Effective target area [Eq. (3)]

$1.58 \mathrm{E}+05$

$5.68 \mathrm{E}-03$

$F \quad$ Impact frequency (per yr) [Eq. (1)]

4.1E-05

takeoffs

8.0E-05

landings

1.2E-04

Total Impact Frequency from Airport Operations (per yr)

commercial + general aviation

1.2E-04 
Table G-2. Nonairport Operations (Overflights)

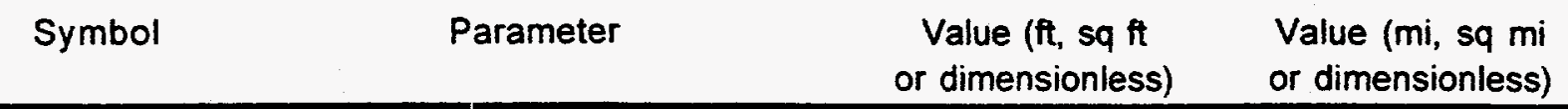

$\operatorname{NPf}(x, y) \quad$ Site-specific crash density rates

(Tables 9 and 10) (crashes per sq $\mathrm{mi}$

per yr)

commercial aviation (air taxi)

3E-06

general aviation aircraft

2E-04

large military

$1 E-07$

small military

$5 E-06$

Effective Area Calculation for Military Aircraft

\begin{tabular}{|c|c|}
\hline WS & $\begin{array}{l}\text { Wingspan (Table 6) } \\
\text { large aircraitt } \\
\text { small aircraft }\end{array}$ \\
\hline $\operatorname{Cot}(\phi)$ & $\begin{array}{l}\text { Cotangent of the crash angle } \\
\text { (Table } 7 \text { ) } \\
\text { large aircrait } \\
\text { small aircraft }\end{array}$ \\
\hline$S$ & $\begin{array}{l}\text { Skid distance (Table 8) } \\
\text { large aircraft } \\
\text { small aircraft }\end{array}$ \\
\hline$A_{f}$ & $\begin{array}{l}\text { Effective fly-in area [Eq. (4)] } \\
\text { large aircraft } \\
\text { small aircraft }\end{array}$ \\
\hline$A_{s}$ & $\begin{array}{l}\text { Effective skid area [Eq. (5)] } \\
\text { large aircraft } \\
\text { small aircraft }\end{array}$ \\
\hline$A_{\text {eff }}$ & $\begin{array}{l}\text { Effective target area [Eq. (3)] } \\
\text { large aircraft } \\
\text { small aircraft }\end{array}$ \\
\hline$F$ & $\begin{array}{l}\text { Impact frequency (per yr) [Eq. (1)] } \\
\text { commercial aviation } \\
\text { general aviation } \\
\text { military (large) } \\
\text { military (small) }\end{array}$ \\
\hline
\end{tabular}

$2.23 E+02$

1.10E+02

7.4

8.4

$7.80 E+02$

$2.46 \mathrm{E}+02$

$2.81 E+05$

1.01E-02

$2.08 E+05$

7.46E-03

$2.66 \mathrm{E}+05$

9.55E-03

$5.62 E+04$

2.02E-03

$5.47 E+05$

1.96E-02

$2.64 E+05$

9.47E-03

4.8E-08

1.1E-06

2. $0 E-09$

4.7E-08

Total Impact Frequency from Nonairport Operations (per yr)

$$
\text { commercial + general aviation + military }
$$

1.2E-06

Total Impact Frequency (pelr yr)

airport + nonairport operations

1.2E-04 
TSFF (TA-21-209) Aircraft Crash Frequency Estimates

Table H-1. Airport Operations (Takeoffs and Landings)

$\begin{array}{lll}\text { Symbol Parameter } \quad \text { Value }(\mathrm{ft}, \mathrm{sq} & \mathrm{ft} & \text { Value }(\mathrm{mi}, \mathrm{sq} \\ \mathrm{mi}\end{array}$ or dimensionless) or dimensionless)

Facility Parameters

$(x, y) \quad$ Orthonormal distance from runway takeoff

landing

$-0.053,-0.28$

$+0.053,+0.28$

$L \quad$ Building length

9.63E+01

$1.82 \mathrm{E}-02$

W Building width

$6.23 E+01$

1.18E-02

$H \quad$ Building height

$7.50 E+01$

1.42E-02

$R \quad$ Building diagonal

$1.15 E+02$

2.17E-02

Commercial Aircraft (Air Taxi) Calculations

$\begin{array}{cl}N & \text { Number of flights per year (takeoffs + landings) } \\ P & \text { Crash rate (Table 1) } \\ & \text { per takeoff } \\ & \text { per landing } \\ & \quad \begin{array}{l}\text { Crash location probability } \\ \text { takeoff (Table 2) }\end{array} \\ & \text { landing (Table 3) } \\ & \text { Wingspan (Table 6) } \\ W S & \text { Cotangent of the crash angle } \\ \text { Cot( } \phi) & \text { (Table 7) } \\ S & \text { Skid distance (Table 8) } \\ A_{f} & \text { Effective fly-in area [Eq. (4)] } \\ A_{s} & \text { Effective skid area [Eq. (5)] } \\ A_{\text {eff }} & \text { Effective target area [Eq. (3)] } \\ F & \text { Impact frequency (per yr) [Eq. (1)] } \\ & \text { takeoffs } \\ & \text { landings } \\ & \text { total }\end{array}$

$3.6 E+03$

1.0E-06

2.3E-06

2.6E- 02

$6.9 \mathrm{E}-03$ 
Table H-1. (cont.)

Symbol

Parameter

Value ( $\mathrm{ft}$, sq $\mathrm{ft}$

Value (mi, sq mi or dimensionless) or dimensionless)

General Aviation Aircraft Calculations

$N \quad$ Number of flights per year (takeoffs + landings)

$8.83 E+03$

$P$

Crash rate (Table 1)

per takeoff

1.1E-05

per landing

2.0E-05

$f(x, y) \quad$ Crash location probability takeoff (Table 4)

1.5E-01

landing (Table 5)

$1.6 \mathrm{E}-01$

WS Wingspan (Table 6)

50

Cot $(\phi)$ Cotangent of the crash angle

8.2

(Table 7)

$S \quad$ Skid distance (Table 8)

60

$A_{f} \quad$ Effective fly-in area [Eq. (4)]

$1.13 E+05$

4.04E-03

$A_{s} \quad$ Effective skid area [Eq. (5)]

$9.88 E+03$

3.54E-04

$A_{\text {eff }} \quad$ Effective target area [Eq. (3)]

$F \quad$ Impact frequency (per yr) [Eq. (1)]

$1.22 E+05$

4. $39 \mathrm{E}-03$

takeoffs

3.2E-05

landings

$6.2 \mathrm{E}-05$

total

9.4E-05

Total Impact Frequency from Airport Operations (per yr)

commercial + general aviation

9.5E-05 
Table H-2. Nonairport Operations (Overflights)

\begin{tabular}{|c|c|}
\hline Symbol & Parameter \\
\hline
\end{tabular}

NPf $(x, y) \quad$ Site-specific crash density rates

(Tables 9 and 10) (crashes per sq $\mathrm{mi}$

per yr)

commercial aviation (air taxi)

3E-06

general aviation aircraft

2E-04

large military

1E-07

small military

5E-06

Effective Area Calculation for Military Aircraft

\begin{tabular}{|c|c|c|}
\hline Ws & $\begin{array}{l}\text { Wingspan (Table 6) } \\
\text { large aircraft } \\
\text { small aircraft }\end{array}$ & $\begin{array}{l}2.23 E+02 \\
1.10 E+02\end{array}$ \\
\hline $\operatorname{Cot}(\phi)$ & $\begin{array}{l}\text { Cotangent of the crash angle } \\
\text { (Table } 7 \text { ) } \\
\text { large aircraft } \\
\text { small aircraft }\end{array}$ & $\begin{array}{l}7 . \\
8 .\end{array}$ \\
\hline$S$ & $\begin{array}{l}\text { Skid distance (Tiable 8) } \\
\text { large aircraft } \\
\text { small aircraft }\end{array}$ & $\begin{array}{l}7.80 E+02 \\
2.46 E+02\end{array}$ \\
\hline$A_{f}$ & $\begin{array}{l}\text { Effective fly-in area [Eq. (4)] } \\
\text { large aircraft } \\
\text { small aircraft }\end{array}$ & $\begin{array}{l}2.17 E+05 \\
1.59 E+05\end{array}$ \\
\hline$A_{s}$ & $\begin{array}{l}\text { Effective skid area [Eq. (5)] } \\
\text { large aircraft } \\
\text { small aircraft }\end{array}$ & $\begin{array}{l}2.63 E+05 \\
5.53 E+04\end{array}$ \\
\hline$A_{\text {eff }}$ & $\begin{array}{l}\text { Effective target area [Eq. (3)] } \\
\text { large aircraft } \\
\text { small aircraft }\end{array}$ & $\begin{array}{l}4.80 \mathrm{E}+05 \\
2.14 \mathrm{E}+05\end{array}$ \\
\hline$F$ & $\begin{array}{l}\text { Impact frequency (per yr) [Eq. (1)] } \\
\text { commercial aviation } \\
\text { general aviation } \\
\text { military (large) } \\
\text { military (small) }\end{array}$ & \\
\hline
\end{tabular}

7.77E-03

5.71E-03

9.45E-03

$1.98 \mathrm{E}-03$

1.72E-02

7.69E-03

4.3E-08

8.8E-07

1.7E-09

3.8E-08

Total Impact Frequency from Nonairport Operations (per yr)

commercial + general aviation + military

9.6E-07

Total Impact Frequency (per yr)

airport + nonairport operations

9.6E-05 


\section{Appendix I}

\section{RLWTF (TA-50-1) Aircraft Crash Frequency Estimates}

Table I-1. Airport Operations (Takeoffs and Landings)

Symbol

Parameter

Value ( $\mathrm{ft}, \mathrm{sq} \mathrm{ft}$

Value (mi, sq mi

or dimensionless) or dimensionless)

Facility Parameters

$(x, y)$ Orthonormal distince from runway takeoff landing

$-1.24,-1.45$

$+1.24,+1.45$

$L \quad$ Building length

$3.10 E+02$

5.87E-02

$W \quad$ Building width

$2.33 E+02$

4.41E-02

$H \quad$ Building height

$6.50 \mathrm{E}+01$

1.23E-02

$R \quad$ Building diagonal

$3.88 \mathrm{E}+02$

7.34E-02

Commercial Aircraft (Air Taxi) Calculations

$\begin{array}{cl}N & \text { Number of flights per year (takeoffs + landings) } \\ P & \begin{array}{c}\text { Crash rate (Table 1) } \\ \text { per takeoff } \\ \text { per landing }\end{array} \\ & \begin{array}{c}\text { Crash location probability } \\ \text { takeoff (Table 2) } \\ \text { landing (Table 3) }\end{array} \\ & \begin{array}{c}\text { Wingspan (Table 6) } \\ W S\end{array} \\ \text { Cot( } \phi) & \begin{array}{l}\text { Cotangent of the crash angle } \\ \text { (Table 7) }\end{array} \\ S & \text { Skid distance (Table 8) } \\ A_{f} & \text { Effective fly-in area [Eq. (4)] } \\ A_{s} & \text { Effective skid area [Eq. (5)] } \\ A_{\text {eff }} & \text { Effective target area [Eq. (3)] } \\ F & \text { Impact frequency (per yr) [Eq. (1)] } \\ & \text { takeoffs } \\ & \text { landings } \\ & \text { total }\end{array}$


Table I-1. (cont.)

Symbol

Parameter

General Aviation Aircraft Calculations

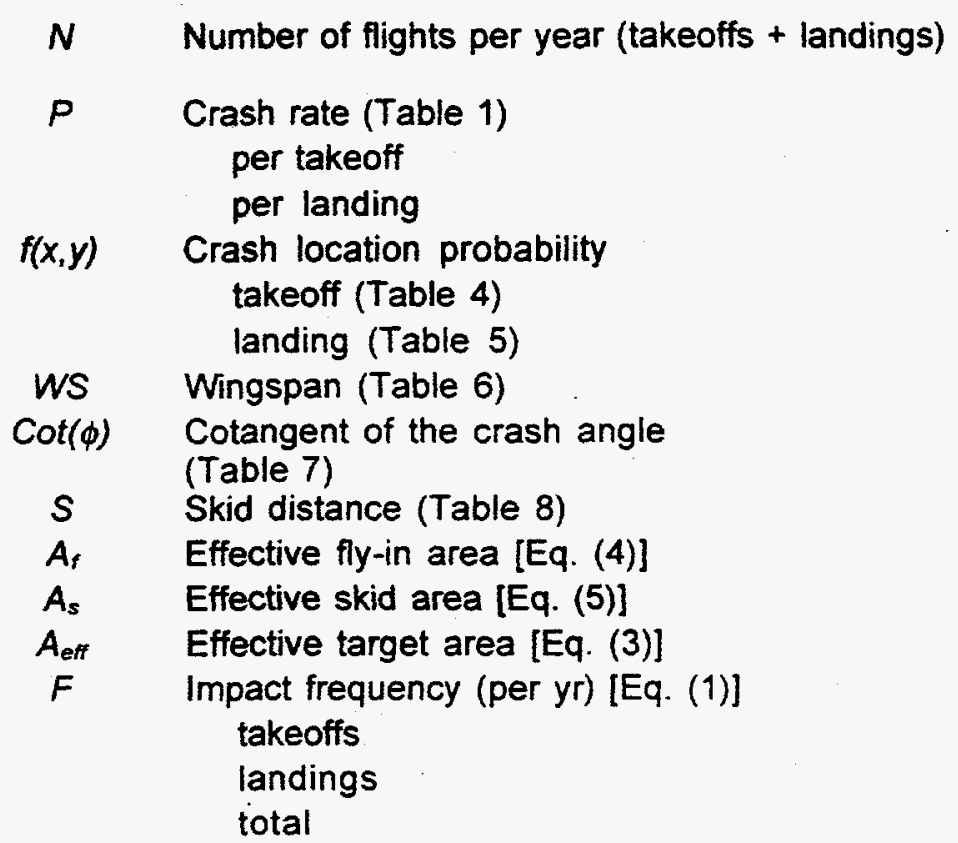

$A_{f} \quad$ Effective fly-in area [Eq. (4)]

$A_{s} \quad$ Effective skid area [Eq. (5)]

$A_{\text {eff }} \quad$ Effective target area [Eq. (3)]

$F \quad$ Impact frequency (per yr) [Eq. (1)]

takeoffs

landings

total

6.7E-07

4.9E-06

5.6E-06

Total Impact Frequency from Airport Operations (per yr)

commercial + general aviation

5.6E-06 
Table 1-2. Nonairport Operations (Overflights)

$\begin{array}{lll}\text { Symbol } & \text { Parameter } & \text { Value }(\mathrm{ft}, \mathrm{sq} \mathrm{ft}\end{array}$ or dimensionless) or dimensionless)

$\operatorname{NPf}(x, y) \quad$ Site-specific crash density rates

(Tables 9 and 10) (crashes per sq mi

per yr)

commercial aviation (air taxi)

3E-06

general aviation aircraft

2E-04

large military

$1 E-07$

small military

$5 E-06$

Effective Area Calculation for Military Aircraft

WS Wingspan (Table 6)

large aircraft

2.23E+02

small aircraft

$1.10 \mathrm{E}+02$

$\operatorname{Cot}(\phi) \quad$ Cotangent of the crash angle

(Table 7)

large aircraft

7.4

small aircraft

8.4

S Skid distance (Table 8)

large aircraft

small aircraf:

$7.80 E+02$

$2.46 E+02$

A, Effective fly-in area [Eq. (4)]

large aircraft

$4.49 E+05$

1.61E-02

small aircraft

$3.85 E+05$

$1.38 \mathrm{E}-02$

$A_{s} \quad$ Effective skid area [Eq. (5)]

large aircraft

4.76E+05

1.71E-02

small aircraft

$1.22 \mathrm{E}+0.5$

4.39E-03

$A_{\text {eff }} \quad$ Effective target area [Eq. (3)]

$9.26 E+05$

3.32E-02

large aircraft

$5.07 E+05$

1.82E-02

F Impact frequency (per yr) [Eq. (1)]

commercial aviation

1.1E-07

general aviation

2.5E-06

military (large)

3.3E-09

military (small)

9.1E-08

Total Impact Frequency from Nonairport Operations (per yr)

commercial + general aviation + military

2.7E-06

Total Impact Frequency (per yr)

airport + nonairport operations

8.3E-06 


\section{Appendix J}

\section{TDF (TA-50-37) Aircraft Crash Frequency Estimates}

\section{Table J-1. Airport Operations (Takeoffs and Landings)}

Symbol

Parameter

Value ( $\mathrm{ft}$, sq ft

Value (mi, sq mi or dimensionless) or dimensionless)

Facility Parameters

$\begin{array}{cl}(x, y) & \begin{array}{c}\text { Orthonormal distance from runway } \\ \text { takeoff } \\ \text { landing }\end{array} \\ L & \text { Building length } \\ W & \text { Building width } \\ H & \text { Building height } \\ R & \text { Building diagorial }\end{array}$

Commercial Aircraft (Air Taxi) Calculations

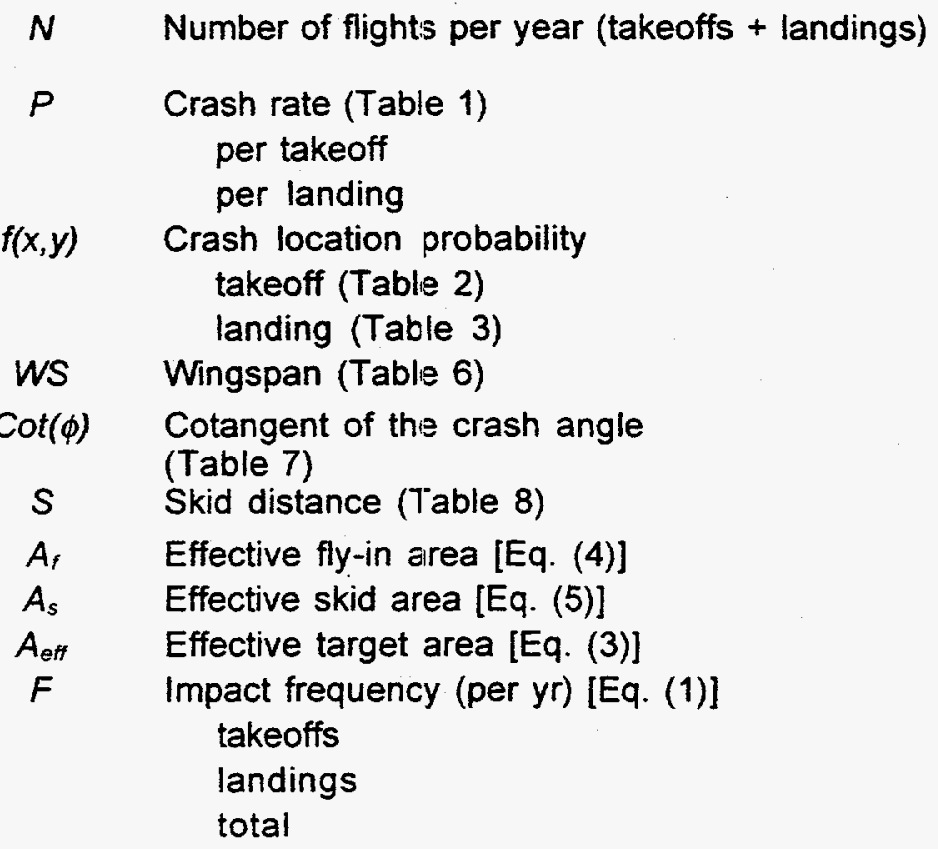


Table J-1. (cont.)

Symbol

Parameter

Value ( $\mathrm{ft}$, sq $\mathrm{ft}$

Value (mi, sq mi

or dimensionless) or dimensionless)

General Aviation Aircraft Calculations

$N \quad$ Number of flights per year (takeoffs + landings)

$8.83 E+03$

$P \quad$ Crash rate (Table 1)

per takeoff

1.1E-05

per landing

2.0E-05

$f(x, y) \quad$ Crash location probability takeoff (Table 4) landing (Table 5)

1.1E-03

4.4E-03

WS Wingspan (Table 6)

50

$\operatorname{Cot}(\phi) \quad$ Cotangent of the crash angle

8.2

(Table 7)

$S \quad$ Skid distance (Table 8)

60

$A_{f} \quad$ Effective fly-in area [Eq. (4)]

$A_{s} \quad$ Effective skid area [Eq. (5)]

$1.13 E+05$

4.04E-03

$A_{\text {eff }} \quad$ Effective target area [Eq. (3)]

$F \quad$ Impact frequency (per yr) [Eq. (1)]

$1.46 E+04$

5.22E-04

1.27E+05

4.56E-03

takeoffs

2.4E-07

landings

$1.8 \mathrm{E}-06$

total

2.0E-06

Total Impact Frequency from Airport Operations (per yr)

commercial + general aviation

2.0E-06 
Table J-2. Nonairport Operations (Overflights)

\begin{tabular}{|c|c|}
\hline Symbol & Parameter \\
\hline
\end{tabular}

$N P f(x, y) \quad$ Site-specific crash density rates

(Tables 9 and 10) (crashes per sq mi

per yr)

commercial aviation (air taxi)

3E-06

general aviation aircraft

2E-04

large military

$1 E-07$

small military

$5 \mathrm{E}-06$

Effective Area Calculation for Military Aircraft

WS Wingspan (Table 6)

large aircraft

$2.23 E+02$

small aircraft

1. $10 E+02$

Cot $(\phi) \quad$ Cotangent of the crash angle

(Table 7)

large aircraft

7.4

small aircraft

\section{4}

S Skid distance (Table 8)

large aircraft

$7.80 E+02$

small aircraft

$2.46 E+02$

Af Effective fly-in area [Eq. (4)]

$1.92 E+05$

$6.88 \mathrm{E}-03$

large aircraft

$1.48 \mathrm{E}+05$

5.29E-03

$A_{s} \quad$ Effective skid area [Eq. (5)]

large aircraft

$3.24 E+05$

1.16E-02

small aircraft

$7.44 E+04$

2.67E-03

$A_{\text {eff }} \quad$ Effective target area [Eq. (3)]

large aircraft

5. $16 \mathrm{E}+05$

1.85E-02

small aircraft

2.22E+05

7.96E-03

F Impact frequency (per yr) [Eq. (1)]

commercial aviation

5.4E-08

general aviation

9.1E-07

military (large)

1.9E-09

military (small)

4. $0 \mathrm{E}-08$

Total Impact Frequency from Nonairport Operations (per yr)

commercial + general aviation + military

1. $0 \mathrm{E}-06$

Total Impact Frequency (per yr)

airport + nonairport operations

3.0E-06 


\section{Appendix K}

\section{WCRRF (TA-50-69) Aircraft Crash Frequency Estimates}

Table K-1. Airport Operations (Takeoffs and Landings)

Symbol

Parameter

Value ( $\mathrm{ft}$, sq $\mathrm{ft}$

Value (mi, sq mi or dimensionless) or dimensionless)

Facility Parameters

$(x, y) \quad$ Orthonormal distance from runway takeoff landing

$L \quad$ Building length

W Building width

$9.00 E+01$

$4.50 E+01$

4.10E+01

$1.01 \mathrm{E}+02$

$-1.37,-1.50$

$+1.37,+1.50$

$H \quad$ Building height

$1.70 \mathrm{E}-02$

$8.52 \mathrm{E}-03$

7.77E-03

1.91E-02

Commercial Aircraft (Air Taxi) Calculations

\begin{tabular}{|c|c|}
\hline$N$ & Number of flights per year (takeoffs + landings) \\
\hline$P$ & $\begin{array}{l}\text { Crash rate (Table 1) } \\
\text { per takeoff } \\
\text { per landing }\end{array}$ \\
\hline$f(x, y)$ & $\begin{array}{l}\text { Crash location probability } \\
\text { takeoff (Table 2) } \\
\text { landing (Table } 3 \text { ) }\end{array}$ \\
\hline WS & Wingspan (Table 6) \\
\hline $\begin{array}{c}\operatorname{Cot}(\phi) \\
S\end{array}$ & $\begin{array}{l}\text { Cotangent of the crash angle } \\
\text { (Table } 7 \text { ) } \\
\text { Skid distance (Table } 8 \text { ) }\end{array}$ \\
\hline$A_{f}$ & Effective fly-in area [Eq. (4)] \\
\hline$A_{s}$ & Effective skid area [Eq. (5)] \\
\hline$A_{\text {eff }}$ & Effective target area [Eq. (3)] \\
\hline$F$ & $\begin{array}{l}\text { Impact frequency (per yr) [Eq. (1)] } \\
\text { takeoffs } \\
\text { landings } \\
\text { total }\end{array}$ \\
\hline
\end{tabular}

$3.6 \mathrm{E}+03$

1.0E-06

2.3E-06

$0.0 E+00$

$0.0 \mathrm{E}+00$

59

10.2

1440

$7.56 \mathrm{E}+04$

2.71E-03

$2.30 E+05$

8.24E-03

$3.05 E+05$

1.10E-02

$0.0 E+00$

$0.0 E+00$

$0.0 E+00$ 
Table K-1. (cont.)

Symbol

Parameter

Value ( $\mathrm{ft}, \mathrm{sq} \mathrm{ft}$

Value (mi, sq mi or dimensionless) or dimensionless)

General Aviation Aircraft Calculations

$N \quad$ Number of flights per year (takeoffs + landings)

$8.83 E+03$

$P \quad$ Crash rate (Table 1)

per takeoff

per landing

1.1E-05

2.0E-05

$f(x, y) \quad$ Crash location probability

takeoff (Table 4)

1.1E-03

landing (Table 5)

4.4E-03

WS Wingspan (Table 6)

$\operatorname{Cot}(\phi) \quad$ Cotangent of the crash angle

8.2

(Table 7)

$S \quad$ Skid distance (Table 8)

60

$A_{f} \quad$ Effective fly-in area [Eq. (4)]

$A_{s} \quad$ Effective skid area [Eq. (5)]

$5.87 \mathrm{E}+04$

$9.04 E+03$

2.11E-03

$A_{\text {eff }} \quad$ Effective target area [Eq. (3)]

$6.78 \mathrm{E}+04$

3.24E-04

2.43E-03

$F \quad$ Impact frequency (per yr) [Eq. (1)]

takeoffs

1.3E-07

landings

9. $4 \mathrm{E}-07$

total

1.1E-06

Total Impact Frequency from Airport Operations (per yr)

commercial + general aviation

1.1E-06 
Table K-2. Nonairport Operations (Overflights)
Symbol
Paramieter
Value ( $\mathrm{ft}$, sq $\mathrm{ft}$
Value (mi, sq mi or dimensionless) or dimensionless)
NPf $(x, y) \quad$ Site-specific crash clensity rates
(Tables 9 and 10) (crashes per sq mi
per yr)
commercial aviation (air taxi)
general aviation aircraft
large military
small military

3E-06

2E-04

$1 \mathrm{E}-07$

$5 \mathrm{E}-06$

Effective Area Calculation for Military Aircraft

\begin{tabular}{|c|c|c|}
\hline WS & $\begin{array}{l}\text { Wingspan (Table 6) } \\
\text { large aircraft } \\
\text { small aircraft }\end{array}$ & $\begin{array}{l}2.23 \mathrm{E}+0 \\
1.10 \mathrm{E}+0\end{array}$ \\
\hline $\operatorname{Cot}(\phi)$ & $\begin{array}{l}\text { Cotangent of the crash angle } \\
\text { (Table } 7 \text { ) } \\
\text { large aircraft } \\
\text { small aircraft }\end{array}$ & $\begin{array}{l}7 . \\
8 .\end{array}$ \\
\hline$S$ & $\begin{array}{l}\text { Skid distance (Table 8) } \\
\text { large aircraft } \\
\text { small aircraft }\end{array}$ & $\begin{array}{l}7.80 \mathrm{E}+\mathrm{C} \\
2.46 \mathrm{E}+\mathrm{C}\end{array}$ \\
\hline$A_{f}$ & $\begin{array}{l}\text { Effective fly-in area [Eq. (4)] } \\
\text { large aircraft } \\
\text { small aircraft }\end{array}$ & $\begin{array}{l}1.20 \mathrm{E}+ \\
8.54 \mathrm{E}+\end{array}$ \\
\hline$A_{s}$ & $\begin{array}{l}\text { Effective skid area [Eq. (5)] } \\
\text { large aircraft } \\
\text { small aircraft }\end{array}$ & $\begin{array}{l}2.52 E+0 \\
5.18 E+0\end{array}$ \\
\hline$A_{\text {eff }}$ & $\begin{array}{l}\text { Effective target area [Eq. (3)] } \\
\text { large aircraft } \\
\text { small aircraft }\end{array}$ & $\begin{array}{l}3.73 \mathrm{E}+ \\
1.37 \mathrm{E}+\end{array}$ \\
\hline$F$ & $\begin{array}{l}\text { Impact frequency (per yr) [Eq. (1)] } \\
\text { commercial aviation } \\
\text { general aviation } \\
\text { military (large) } \\
\text { military (small) }\end{array}$ & \\
\hline
\end{tabular}

9.05E-03

$1.86 \mathrm{E}-03$

1.34E-02

4.92E-03

3.3E-08

4.9E-07

1.3E-09

2.5E-08

Total Impact Frequency from Nonairport Operations (per yr)

commercial + general aviation + military

5.4E-07

Total Impact Frequency (per yr)

airport + nonairport operations

1.6E-06 


\section{Area G (TA-54-G) Aircraft Crash Frequency Estimates}

Table L-1. Airport Operations (Takeoffs and Landings)

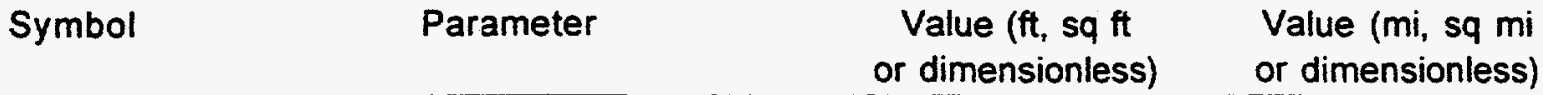

\section{Facility Parameters}

$(x, y) \quad$ Orthonormal distance from runway takeoff landing

$L \quad$ Building length

$3.20 E+02$

$2.46 E+02$

$3.80 E+01$

H Building height

4.04E+02

$+1.63,-2.06$

$-1.63,+2.06$

$R \quad$ Building diagonal

Commercial Aircraft (Air Taxi) Calculations

$\begin{array}{cl}N & \text { Number of flights per year (takeoffs + landings) } \\ P & \text { Crash rate (Table 1) } \\ & \text { per takeoff } \\ & \text { per landing } \\ & \text { Crash location probability } \\ f(x, y) & \text { takeoff (Table 2) } \\ & \quad \text { landing (Table } 3 \text { ) } \\ \text { WS } & \text { Wingspan (Table 6) } \\ \text { Cot }(\phi) & \text { Cotangent of the crash angle } \\ S & \text { (Table 7) } \\ A_{f} & \text { Skid distance (Table 8) } \\ A_{s}: & \text { Effective fly-in area [Eq. (4)] } \\ A_{\text {eff }} & \text { Effective skid area [Eq. (5)] } \\ F & \text { Effective target area [Eq. (3)] } \\ & \text { Impact frequency (per yr) [Eq. (1)] } \\ & \text { takeoffs } \\ & \text { landings } \\ & \text { total }\end{array}$


Table L-1. (cont.)

Symbol

Parameter

Value ( $\mathrm{ft}$, sq ft

Value (mi, sq mi

or dimensionless) or dimensionless)

General Aviation Aircraft Calculations

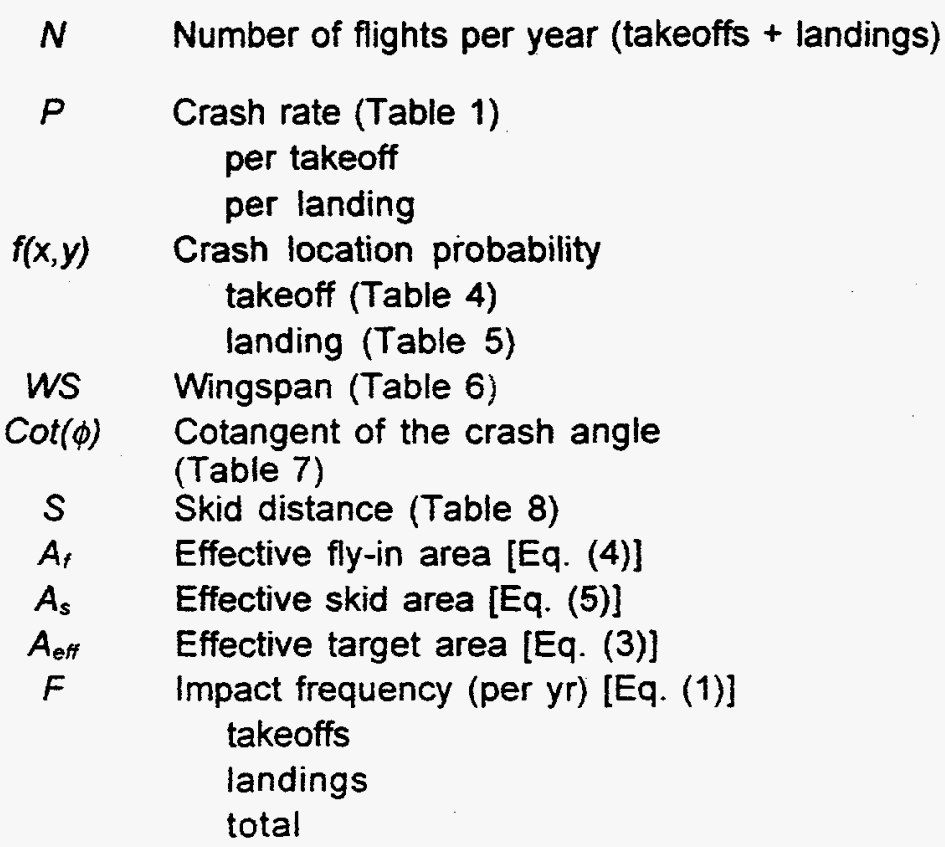

Total Impact Frequency from Airport Operations (per yr)

$$
\text { commercial + general aviation }
$$

1.7E-06 
Table L-2. Nonairport Operations (Overflights)

\begin{tabular}{|c|c|c|c|}
\hline Symbol & Parameter & $\begin{array}{l}\text { Value ( } \mathrm{ft} \text {, sq } \mathrm{ft} \\
\text { or dimensionless) }\end{array}$ & $\begin{array}{l}\text { Value (mi, sq mi } \\
\text { or dimensionless) }\end{array}$ \\
\hline
\end{tabular}

$\operatorname{NPf}(x, y) \quad$ Site-specific crash density rates

(Tables 9 and 10) (crashes per sq mi

per yr)

commercial aviation (air taxi)

3E-06

general aviation aircraft

2E-04

large military

$1 E-07$

small military

$5 E-06$

Effective Area Calculation for Military Aircraft

\begin{tabular}{|c|c|c|c|}
\hline WS & Wingspan (Table $(i)$ & & \\
\hline & large aircraft & $2.23 E+02$ & \\
\hline & small aircraft & $1.10 E+02$ & \\
\hline $\operatorname{Cot}(\phi)$ & $\begin{array}{l}\text { Cotangent of the crash angle } \\
\text { (Table 7) }\end{array}$ & & \\
\hline & large aircraft & 7.4 & \\
\hline & small aircraft & 8.4 & \\
\hline$S$ & Skid distance (Table 8) & & \\
\hline & large aircraft & $7.80 E+02$ & \\
\hline & small aircraft & $2.46 E+02$ & \\
\hline$A_{f}$ & Effective fly-in area [Eq. (4)] & & \\
\hline & large aircraft & $3.42 E+05$ & $1.23 E-02$ \\
\hline & small aircraft & $2.86 E+05$ & $1.02 E-02$ \\
\hline$A_{s}$ & Effective skid area [Eq. (5)] & & \\
\hline & large aircraft & $4.89 E+05$ & $1.75 E-02$ \\
\hline & small aircraft & $1.26 \mathrm{E}+05$ & 4.53E-03 \\
\hline$A_{\text {eff }}$ & Effective target area [Eq. (3)] & & \\
\hline & large aircraft & 8.31E+05 & $2.98 E-02$ \\
\hline & small aircraft & $4.12 E+05$ & $1.48 \mathrm{E}-02$ \\
\hline$F$ & Impact frequency (per yr) [Eq. (1)] & & \\
\hline & commercial aviation & & 1.0E-07 \\
\hline & general aviation & & $1.9 \mathrm{E}-06$ \\
\hline & military (large) & . & $3.0 \mathrm{E}-09$ \\
\hline & military (small) & & $7.4 E-08$ \\
\hline
\end{tabular}

Total Impact Frequency from Nonairport Operations (per yr)

commercial + general aviation + military

2.1E-06

Total Impact Frequency (per yr)

airport + nonairport operations

3.8E-06 


\section{Appendix M}

\section{Plutonium Facility (TA-55-4) Aircraft Crash Frequency Estimates}

Table M-1. Airport Operations (Takeoffs and Landings)

\begin{tabular}{|c|c|c|c|}
\hline Symbol & Parameter & $\begin{array}{c}\text { Value ( } \mathrm{ft}, \mathrm{sq} \mathrm{ft} \\
\text { or dimensionless) }\end{array}$ & $\begin{array}{l}\text { Value (mi, sq mi } \\
\text { or dimensionless) }\end{array}$ \\
\hline
\end{tabular}

Facility Parameters
$(x, y) \quad$ Orthonormal distance from runway takeoff landing

$L \quad$ Building length

$2.84 \mathrm{E}+02$

W Building width

$2.62 E+02$

$3.20 E+01$

$3.86 \mathrm{E}+02$

$-1.49,-1.42$

$+1.49,+1.42$

$H \quad$ Building height

$5.38 \mathrm{E}-02$

4.96E-02

$R \quad$ Building diagonal

$6.06 \mathrm{E}-03$

7.32E-02

Commercial Aircraft (Air Taxi) Calculations

$\begin{array}{cl}N & \text { Number of flights per year (takeoffs + landings) } \\ P & \text { Crash rate (Table 1) } \\ & \text { per takeoff } \\ & \text { per landing } \\ & \text { Crash location probability } \\ & \text { takeoff (Table 2) } \\ f(x, y) & \text { landing (Table 3) } \\ & \text { Wingspan (Table 6) } \\ W S & \text { Cotangent of the crash angle } \\ \text { Cot( } \phi) & \text { (Table 7) } \\ S & \text { Skid distance (Table 8) } \\ A_{f} & \text { Effective fly-in area [Eq. (4)] } \\ A_{s} & \text { Effective skid area [Eq. (5)] } \\ A_{\text {eff }} & \text { Effective target area [Eq. (3)] } \\ F & \text { Impact frequency (per yr) [Eq. (1)] } \\ & \text { takeoffs } \\ & \text { landings } \\ & \text { total }\end{array}$

$0.0 E+00$

$0.0 E+00$ 
Table M-1. (cont.)

Symbol

Parameter

Value $(\mathrm{ft}$, sq $\mathrm{ft}$

Value (mi, sq mi

General Aviation Aircraft Calculations

$\begin{array}{cl}N & \text { Number of flights per year (takeoffs + landings) } \\ P & \text { Crash rate (Table 1) } \\ & \text { per takeoff } \\ & \text { per landing } \\ & \text { Crash location probability } \\ f(x, y) \quad \text { takeoff (Table 4) } & \text { landing (Table 5) } \\ & \text { Wingspan (Table 6) } \\ \text { WS } & \text { Cotangent of the crash angle } \\ \text { Cot( } \phi) & \text { (Table 7) } \\ S & \text { Skid distance (Table 8) } \\ A_{f} & \text { Effective fly-in area [Eq. (4)] } \\ A_{s} & \text { Effective skid area [Eq. (5)] } \\ A_{\text {eff }} & \text { Effective target area [Eq. (3)] } \\ F & \text { Impact frequency (per yr) [Eq. (1)] } \\ & \text { takeoffs } \\ & \text { landings } \\ & \text { total }\end{array}$

Total Impact Frequency from Airport Operations (per yr) 
Table M-2. Nonairport Operations (Overflights)

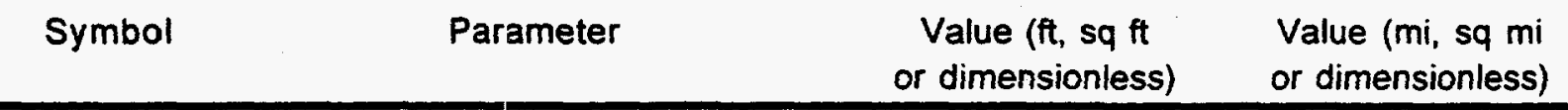

NPf $(x, y) \quad$ Site-specific crash density rates

(Tables 9 and 10) (crashes per sq mi

per yr)

commercial aviation (air taxi)

3E-06

general aviation aircraft

2E-04

large military

$1 E-07$

small military

5E-06

Effective Area Calculation for Military Aircraft

WS Wingspan (Table 6)

large aircraft

small aircraft

$2.23 E+02$

$1.10 E+02$

Cot $(\phi)$ Cotangent of the crash angle

(Table 7)

large aircraft

small aircraft

7.4

8.4

S Skid distance (Table 8)

large aircraft

$7.80 E+02$

small aircraft

$2.46 \mathrm{E}+02$

$A_{f} \quad$ Effective fly-in area [Eq. (4)]

large aircraft

$3.05 E+05$

1.09E-02

small aircraft

$2.50 E+05$

8.97E-03

$A_{s} \quad$ Effective skid area [Eq. (5)]

large aircraft

$4.75 \mathrm{E}+05$

1.71E-02

small aircraft

$A_{\text {eff }} \quad$ Effective target area [Eq. (3)]

$1.22 E+05$

4.38E-03

large aircraft

$7.80 E+05$

2.80E-02

small aircraft

$3.72 E+05$

$1.34 \mathrm{E}-02$

F Impact frequency (per yr) [Eq. (1)]

commercial aviation

9.5E-08

general aviation

1.7E-06

military (large)

2. $8 \mathrm{E}-09$

military (small)

6.7E-08

Total Impact Frequency from Nonairport Operations (per yr)

$$
\text { commercial + general aviation + military }
$$

Total Impact Frequency (per yr)

$$
\text { airport + nonairport operations }
$$

5.6E-06 